\title{
ON NEIGHBOURLY TRIANGULATIONS
}

\author{
BY
}

K. S. SARKARIA

\begin{abstract}
A simplicial complex is called $d$-neighbourly if any $d+1$ vertices determine a $d$-simplex. We give methods for constructing 1-neighbourly triangulations of 3- and 4-manifolds; further we discuss some relationships between $d$ neighbourly triangulations, chromatic numbers and the problem of finding upper and lower bounds on the number of simplices and locating the zeros of the characteristic polynomial of a triangulation. A triangulation of an orientable manifold is called order-orientable if there exists some ordering of the vertices which orients the manifold. We give necessary conditions for their existence; also we construct such triangulations on 3-dimensional handlebodies and discuss the problem of recognising finite monotone subsets of an affine space by using these ideas.
\end{abstract}

(1.1) A (finite, abstract) simplical complex $K$ will be called $d$-neighbourly if any $d+1$ vertices of $K$ are the vertices of some $d$-simplex of $K$; instead of 1-neighbourly we will simply say neighbourly. A space $X$ will be called triangulable, and said to admit a triangulation $K$, if $X$ is homeomorphic to the geometrical realization $|K|$ of a simplicial complex $K$. The word manifold will always refer to a 'compact, Hausdorff manifold' (with or without boundary); we remark that for dimensions $\geqslant 4$ it is still not known whether all such manifolds are triangulable: so, in these dimensions, the additional (possibly vacuous) hypothesis of triangulability will be assumed.

(1.1.1) In 1911 Carathéodory [4] discovered the fact that the $n$-sphere $S^{n}$ admits an $[(n-1) / 2]$-neighbourly triangulation $C_{n}^{x}$ having $x$ vertices, where $x$ is any integer $\geqslant n+2$. (However the combinatorial structure of these 'cyclic triangulations' was understood only after their rediscovery by Gale [10, 11] and Motzkin [22]; in (4.1) the reader will find a definition of $C_{n}^{x}$ based on 'Gale's evenness condition', and in (4.1.1) we verify, without using any convexity notion, that $C_{n}^{x}$ in fact triangulates $S^{n}$.) It is natural to enquire whether an analogous phenomenon is exhibited by other $n$-manifolds $M^{n}$. It is clear that one would have to assume that the homotopy groups $\pi_{i}\left(M^{n}\right)$ are trivial for $i<[(n-1) / 2]$; we conjecture (see (6.1.3)) that these conditions are in fact also sufficient. In this context for 3-manifolds we have been able to prove the following general theorem.

NEIGHBOURLINESS THEOREM FOR 3-MANIFOLDS. If $M^{3}$ is any connected 3-manifold which is either closed or with a nonempty connected boundary, then there exists an integer $\nu$ such that for all $x \geqslant \nu, M^{3}$ admits a neighbourly triangulation $K^{x}$ having $x$ vertices.

Received by the editors September 21, 1981 and, in revised form, March 17, 1982.

1980 Mathematics Subject Classification. Primary 57Q15; Secondary 52A40, 05C15. 
The proof is given in $\S 3$ : see (3.2.1) and (3.2.5). The reader can, if he so wishes, read $\$ 3$ before $\S 2$. We use a theorem of Bing [2] which ensures that every connected 3-manifold with nonempty boundary has a triangulation all of whose vertices lie on the boundary. The idea of the proof is essentially to improve such a triangulation step by step till it becomes neighbourly.

(1.1.1a) AdDED MARCH, 1982. The above theorem resolves, in the affirmative, 'an open question' mentioned on p. 419 of the 1977 paper of Altshuler [30]. But it turns out that the neighbourliness of 3-manifolds was one of the results already proved in the remarkable 1970 paper of Walkup [38, Theorem 4, p. 76 and pp. 89-91]. Walkup's proof can be easily adapted to show that in fact all connected 3-pseudomanifolds are neighbourly. We present our construction of neighbourly triangulations because it is quite different, and perhaps geometrically more satisfying, than Walkup's.

(1.1.2) We remark that the method for proving the above theorem can, in some special cases, be adapted to give neighbourly triangulations with a reasonable number of vertices. However in general there is very little control on the number of vertices. Therefore it is of interest to look at another method which enables one to give explicit neighbourly triangulations for some cartesian products. In this method the starting point is the 'map colour theorem' of Ringel et al. [24] which furnishes us with an infinite number of orientable (or nonorientable) closed manifolds $M^{2}$ which admit a neighbourly triangulation: such a triangulation has $x_{M}$ vertices where the integer $x_{M}$ is uniquely determined by $\operatorname{dim} H_{1}\left(M^{2}, \mathbf{Z}_{2}\right)$ in a simple way; see (2.1.1) and (6.1.1). Using such 'neighbourly' 2-manifolds we are able to deduce the following result.

THEOREM. If $M^{2}$ and $N^{2}$ are neighbourly closed 2-manifolds and $M^{2}$ is orientable, then $M^{2} \times S^{1}$ (resp. $M^{2} \times N^{2}$ ) admits a neighbourly triangulation with 3. $x_{M}$ (resp. $\left.x_{M} \cdot x_{N}\right)$ vertices.

This theorem will be proved in $\S 2$ : see (2.2) and (2.3).

(1.1.3) Let us denote by $\alpha_{i}(K)$ the number of $i$-simplices in a simplicial complex $K$. We recall that Stanley [28] proved that if $K$ is any triangulation of $S^{n}$ having $x$ vertices, then $\alpha_{i}(K) \leqslant \alpha_{i}\left(C_{n}^{x}\right)$ for all $i$. (We note that before Stanley's work, McMullen [19, 20] had proved these inequalities in case $K$ is a 'convex' triangulation of $S^{n}$; i.e. when $K$ can be realised as the boundary of an $n+1$ dimensional convex polytope.) If $K$ is a triangulation of some other manifold $M^{n}$ (e.g. if $K$ is a neighbourly triangulation of the torus or of $S^{2} \times S^{2}$ ), then these inequalities may be false. However Stanley's theorem can be reformulated in terms of the ' $h$-vector' (see, [28, p. 137]) and it is unknown whether this version of his theorem holds for all manifolds. For more on upper and lower bounds see (6.1.2)-(6.1.5).

(1.1.4) In $\$ 4$ we introduce the new concept of an order-orientable triangulation, i.e. a triangulation of an oriented manifold whose vertices can be totally ordered in such a way that the induced orientations of the top dimensional simplices orient the manifold. As one would expect such triangulations are not very common: if a manifold $M^{n}$ admits an order-orientable triangulation, then $\partial M^{n}$ is nonempty and 
the inclusion $\partial M^{n} \rightarrow M^{n}$ induces an isomorphism in homology for dimensions < $[(n-2) / 2](4.2 .3)$. This is an immediate consequence of the following result which is proved in (4.2.2).

AN ORIENTATION LEMMA. If $M^{n}$ has a triangulation $K$ whose vertices are labelled as $1,2, \ldots, x$ in accordance with a total ordering which orients $M^{n}$, then (int $\left.K\right) \cap C_{n-1}^{x}$ $=\varnothing$.

It is important to point out, however, that order-orientable triangulations are abundant enough to justify their study; e.g., we prove in $(4.2 .5)(\mathrm{c})$ that every three-dimensional handlebody admits an order-orientable triangulation. See also (6.1.6).

The only place where convexity plays a key role is in subsection (4.3). Let $A^{n}$ be a real affine space and $X \subseteq A^{n}$ a finite subset whose $x(\geqslant n+1)$ points are in general position; denote by $K_{X}$ the simplicial complex which arises from the boundary facets of the convex hull of $X$. As an application of the orientation lemma, we give in (4.3.2) a new proof of the fact that if $X$ admits a monotone sequence, then $K_{X} \cong C_{n-1}^{x}$. (A 'monotone sequence' is a total ordering of $X$ under which any $n+1$ points of $X$ determine the same orientation of $A^{n}$; the usual proof of this presumably known result would follow the lines of (6.2.4).) Using this result we describe in (4.3.4) and (4.3.5) a practical method for deciding whether a given $X \subseteq A^{n}$ admits a monotone sequence; this answers a question which has been raised by Uhrin [29] and H. Gupta (oral communication): see (4.3.3). The reader will find in (6.2) a short account of the history of monotone sequences.

(1.1.5) The ith chromatic number $\operatorname{ch}_{i}(X)$ of a triangulable space is the smallest number of colours which can be assigned to the $i$-simplices of any triangulation of $X$ in such a way that not all the faces of an $i+1$ simplex have the same colour. This definition was given in [25]. We remark that $\operatorname{ch}_{0}\left(M^{2}\right)$ are precisely the classical chromatic numbers and thus the 'map colour theorem' of Ringel et al. and the 'four colour theorem' of Appel and Haken are equivalent to calculating $\operatorname{ch}_{0}\left(M^{2}\right)$ for all closed 2-manifolds.

Calculation of SOME Chromatic NUmbers. (a) If the triangulable space $X$ has dimension $\geqslant 2 i+3$, then $\operatorname{ch}_{i}(X)=\infty$. (b) If $M^{n}$ is a closed manifold and $n \geqslant 2$, then $\operatorname{ch}_{n-1}\left(M^{n}\right)=2$.

This theorem is proved in $\S 5$ : see (5.1.3) and (5.2.1). We note that the cyclic triangulations $C_{n}^{x}$ are used in the proof of (a) and that the two colour thoerem $\operatorname{ch}_{1}\left(M^{2}\right)=2$ is used in the constructions of $\S 2$.

In [25] a simple upper bound (involving $\operatorname{dim} H_{n-1}\left(M^{n} ; \mathbf{Z}_{2}\right)$ ) is given for the codimension 2 chromatic numbers $\mathrm{ch}_{n-2}\left(M^{n}\right)$ for any pseudomanifold $M^{n}, n \geqslant 2$. If $M^{n}$ is a manifold and $n \geqslant 3$, then we can prove that $\mathrm{ch}_{n-2}\left(M^{n}\right) \leqslant 4$. We hope to give in another paper a proof of the fact that the chromatic numbers $\operatorname{ch}_{i}\left(M^{n}\right)$, $n \leqslant 2 i+2$, are finite.

(1.2) As far as possible the notation conforms to standard topological practice. We think of a simplicial complex $K$ primarily as a combinatorial object (i.e. a finite set of finite sets with the property that $\sigma \in K, \theta \subseteq \sigma$ implies $\theta \in K$ ) and secondarily as 
a topological object (i.e. a subdivided topological space which is a homeomorph of some geometrical realization of $K$ ). Many times, when no confusion seems possible, we have adopted the standard convention of using the same letter $K$ to denote the topological object; occasionally it is denoted by $|K|$. Likewise the letter $\sigma$ or the notation $\{a, b, c, d\}$, etc. may stand both for the abstract finite set and the corresponding closed topological simplex. (In (4.3) the realizations $|K|$ will be rectilinear and in a specified affine space.) If simplices $\sigma$ and $\theta$ have distinct vertices the simplex $\sigma \cup \theta$ is also denoted by $\sigma . \theta$ (their 'join'); the join of 2 simplicial complexes $K$ and $L$, which have distinct vertices, is the complex $K . L$ consisting of all simplices $\sigma . \theta, \sigma \in K, \theta \in L$. If $\sigma \in K$ the 'link' of $\sigma$ in $K$ consists of all $\theta \in K$ s.t. $\sigma . \theta \in K$; it is denoted by $\mathrm{Lk}_{K} \sigma$ or just $\mathrm{Lk} \sigma$. The 'open star' of $\sigma$ in $K$ consists of all $\theta \in K$ s.t. $\sigma \subseteq \theta$; it is denoted by $\mathrm{St}_{K} \sigma$ or just St $\sigma$. The open subset of $|K|$ determined by the interiors of all simplices of $\mathrm{St}_{K} \sigma$ is denoted by $\left|\mathrm{St}_{K} \sigma\right|$. We denote by $\overline{\mathrm{St}_{K} \sigma}$ the 'closed star' of $\sigma$, i.e. the subcomplex of $K$ generated by $\mathrm{St}_{K} \sigma$. Superscripts to simplices or triangulable spaces denote their dimension. Sometimes a simplicial complex is specified by just enumerating its top dimensional simplices.

We have adopted the convention of stating a result for manifolds even when the proof involved uses this concept in a (very) mild way; in all these cases the corresponding generalization (to 'pseudomanifolds', 'Euler spaces', etc.) is transparent.

ACKNowledgements. I am grateful to Professor H. Gupta and to Dr. M. V. Nori for invaluable assistance given towards the preparation of this paper. I would also like to thank Professors Fr. Fabricius-Bjerre and Richard Stanley for sending copies of some papers which were unavailable to me. I am indebted to the referee for pointing out a number of mistakes which had crept into the original version of this paper and for suggesting various improvements in the exposition.

\section{Neighbourly triangulations on products.}

(2.1) A neighbourly space will be one which admits a neighbourly triangulation. The circle is neighbourly and it admits a unique neighbourly triangulation, viz. the one with 3 vertices. If a closed connected 2-manifold $M$ admits a neighbourly triangulation with $x$ vertices then its Euler characteristic is

$$
x-\left(\begin{array}{l}
x \\
2
\end{array}\right)+\frac{2}{3}\left(\begin{array}{l}
x \\
2
\end{array}\right)=2-\operatorname{dim} H_{1}\left(M ; \mathbf{Z}_{2}\right)
$$

hence $\operatorname{dim} H_{1}\left(M ; \mathbf{Z}_{2}\right)=(x-4)(x-3) / 6$. This proves the 'only if' part of the following theorem. The converse is much more difficult to prove and is the result of the combined efforts of Heffter (1891), Ringel (1954, 1961), Gustin (1963), Terry, Welch and Youngs (1963) and many others. (See Ringel [24] for a proof and for references to the original sources; neighbourly triangulations correspond to the "triangular imbeddings" of this book.)

(2.1.1) $A$ closed connected 2-manifold $M$ admits a neighbourly triangulation with $x$ vertices if and only if $x \geqslant 4$ and $\operatorname{dim} H_{1}\left(M ; \mathbf{Z}_{2}\right)=(x-4)(x-3) / 6$. 
This theorem has the following immediate corollary:

(2.1.2) There exist an infinite number of orientable (or nonorientable) closed 2-manifolds which are neighbourly.

For each neighbourly 2-manifold $M$ the number $x$ of (2.1.1) is uniquely determined by $M$; henceforth it will be denoted by $x_{M}$.

(2.2) If the closed orientable 2-manifold $M^{2}$ is neighbourly, then $M^{2} \times S^{1}$ admits a neighbourly triangulation with 3. $x_{M}$ vertices.

(2.2.1) We equip $M^{2}$ with an orientation and with a neighbourly triangulation $L$; we observe that the orientation of $M^{2}$ fixes an orientation on each 2-simplex of $L$. Next we note that given any triangulation of a closed 2-manifold one can assign colours 0 and 1 to its edges in such a way that no triangle has all three edges of the same colour. (A result more general than this will be proved in (5.2.1); the only prerequisite for understanding its proof is (5.1).) Using this lemma we assign such a 'good' 2-colouring to the edges of $L$. Using this data we will give a canonical construction for a neighbourly triangulation $K$ of $M^{2} \times S^{1}$; the vertices of $K$ will be the ordered pairs $i \alpha$ where $i$ is a vertex of $L$ and $\alpha=1,2$ or 3 .

(2.2.2) For each 2-simplex $\sigma^{2} \in L$ we will define in a canonical way a triangulation $K_{\sigma}$ of the solid torus $\sigma^{2} \times S^{1} ; K_{\sigma}$ will be isomorphic to the triangulation shown in Figure 1 (where the two triangles on the left and right are assumed identified). The vertices of $K_{\sigma}$ will be $i \alpha, i \in \sigma^{2}, 1 \leqslant \alpha \leqslant 3$. Corresponding to each vertex $i$ of $\sigma^{2}$ there will be three 'vertical edges' in $K_{\sigma}$, viz. $\{i 1, i 2\},\{i 2, i 3\}$ and $\{i 3, i 1\}$ and corresponding to each edge $\{i, j\}$ of $\sigma^{2}$ there will be three 'horizontal edges' in $K_{\sigma}$, viz. $\{i 1, j 1\},\{i 2, j 2\}$ and $\{i 3, j 3\}$. Further corresponding to each edge $\{i, j\}$ of $\sigma^{2}$, where $i$ precedes $j$ with respect to the orientation of $\sigma^{2}$, there will also be three 'sloping edges' in $K_{\sigma}$; if $\{i, j\}$ has colour 0 (resp. 1) then these edges will be $\{i 1, j 2\}$, $\{i 2, j 3\}$ and $\{i 3, j 1\}$ (resp. $\{i 2, j 1\},\{i 3, j 2\}$ and $\{i 1, j 3\}$ ).



FIGURE 1

(2.2.3) Let $\sigma^{1}$ be any 1 -simplex of $L$; it is incident to precisely two 2-simplices $\sigma_{1}^{2}$, $\sigma_{2}^{2}$ of $L$. Let $\sigma^{1}=\{i, j\}$; if $i$ precedes (resp. follows) $j$ with respect to the orientation of $\sigma_{1}^{2}$ then $i$ follows (resp. precedes) $j$ with respect to the orientation of $\sigma_{2}^{2}$. So by adding the three new tetrahedra $\{i 1, j 1, i 2, j 2\},\{i 2, j 2, i 3, j 3\}$ and $\{i 3, j 3, i 1, j 1\}$ to $K_{\sigma_{1}} \cup K_{\sigma_{2}}$ we get a triangulation of (a space homeomorphic to) $\left(\sigma_{1} \cup \sigma_{2}\right) \times S^{1}$. We continue this process till all the 1-simplices of $L$ are exhausted; this gives us the required neighbourly triangulation $K$ of $M^{2} \times S^{1}$. 
(2.3) If $M^{2}$ and $N^{2}$ are closed neighbourly 2-manifolds of which one is orientable, then $M^{2} \times N^{2}$ admits a neighbourly triangulation with $x_{M} \cdot x_{N}$ vertices.

(2.3.1) We assume that $M^{2}$ is orientable and equip it with an orientation. Furthermore $M^{2}$ and $N^{2}$ are equipped with neighbourly triangulations $L_{1}$ and $L_{2}$, respectively; the edges of $L_{1}$ are equipped with a 'good' 2-colouring as in (2.2.1) and the vertices of $L_{2}$ are totally ordered and accordingly assigned the numbers $1,2, \ldots, x_{N}$. Using this data we will construct a neighbourly triangulation $K$ on $M^{2} \times N^{2}$; the vertices of $K$ will be all ordered pairs $i \alpha$ where $i$ is a vertex of $L_{1}$ and $1 \leqslant \alpha \leqslant x_{N}$.

(2.3.2) For each 2-simplex $\sigma^{2} \in L_{1}$ we will define in a canonical way a triangulation $K_{\sigma}$ for the manifold $\sigma^{2} \times N^{2}$. The vertices of $K_{\sigma}$ will be $i \alpha, i \in \sigma^{2}, 1 \leqslant \alpha \leqslant x_{N}$. Corresponding to each edge $\sigma^{1}=\{i, j\}$ of $\sigma^{2}$, where $i$ precedes $j$ with respect to the orientation of $\sigma^{2}$, and an edge $\theta^{1}=\{\alpha, \beta\}, \alpha<\beta$, of $L_{2}$, the rectangle $\sigma^{1} \times \theta^{1}$ will be equipped with the triangulation of Figure $2 \mathrm{a}$ (resp. Figure $2 \mathrm{~b}$ ) if $\sigma^{1}$ has colour 0 (resp. 1).



Figure 2a



FIgURE 2b

For each 2-simplex $\theta^{2} \in L_{2}$ one has a 4-cell $\sigma^{2} \times \theta^{2}$ and within its boundary there are nine rectangles of the above type; these nine rectangles constitute a 2-torus. By means of the construction just given we have equipped this torus with a triangulation isomorphic to that shown in Figure 3a.

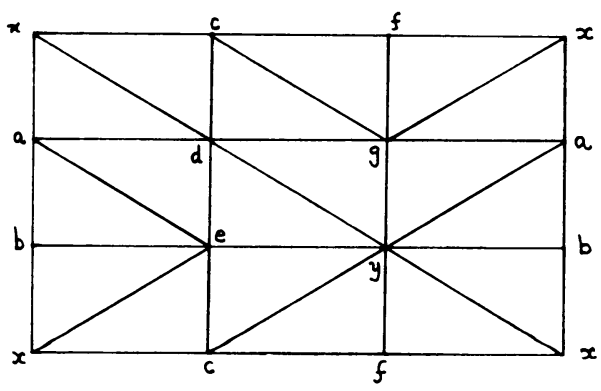

Figure 3a

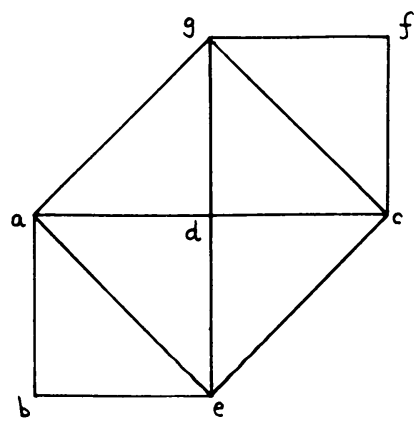

FIGURE 3b

Such a toral triangulation has exactly 2 vertices $x$ and $y$ which are joined to all other vertices by 1 -simplices. Using the remaining 7 vertices one constructs a triangulation of the 2-disk as shown in Figure 3b. We will equip the 4-cell $\sigma^{2} \times \theta^{2}$ with the triangulation obtained by taking the join of the 1-simplex $\{x, y\}$ and the simplicial complex of Figure 3b. This completes the description of $K_{\sigma}$. 
(2.3.3) Let $\sigma^{1}$ be any 1-simplex of $L_{1}$; it is incident to precisely two 2-simplices $\sigma_{1}^{2}$, $\sigma_{2}^{2}$ of $L_{1}$. Let $\sigma^{1}=\{i, j\}$; if $i$ precedes (resp. follows) $j$ with respect to the orientation of $\sigma_{1}^{2}$, then $i$ follows (resp. precedes) $j$ with respect to the orientation of $\sigma_{2}^{2}$. If a rectangle $\sigma^{1} \times \theta^{1}$ occurs in one of the bounding manifolds $\partial\left(\sigma_{1}^{2} \times N^{2}\right)$ and $\partial\left(\sigma_{2}^{2} \times N^{2}\right)$ with the triangulation of Figure $2 \mathrm{a}$ then it will occur in the other with the triangulation of Figure $2 \mathrm{~b}$. We 'patch' these two together by adding, for each such rectangle, a new tetrahedron $\{i \alpha, j \alpha, i \beta, j \beta\}$. In this way we get a space which is homeomorphic to $\left(\sigma_{1}^{2} \cup \sigma_{2}^{2}\right) \times N^{2}$ minus as many open 4-cells as there are 3-cells of the type $\sigma^{1} \times \theta^{2}$ in $\sigma_{1}^{2} \times N^{2}$ (or $\sigma_{2}^{2} \times N^{2}$ ). Now we will 'fill' the $\theta$ th hold as follows.

We examine the triangulation $T_{\theta}$ of its boundary. Firstly three tetrahedra will be contributed to it by $\sigma^{1} \times \theta^{2}$ seen as a subspace of $\partial\left(\sigma_{1}^{2} \times N^{2}\right)$; this subcomplex is isomorphic to the one shown in Figure 4a. The three tetrahedra contributed by $\sigma^{1} \times \theta^{2}$ seen as a subspace of $\partial\left(\sigma_{2}^{2} \times N^{2}\right)$ will now be as in Figure $4 \mathrm{~b}$. Besides this three more tetrahedra, viz. $\{1,2,3,4\},\{3,4,5,6\}$ and $\{5,6,1,2\}$, are contributed by the patching process.

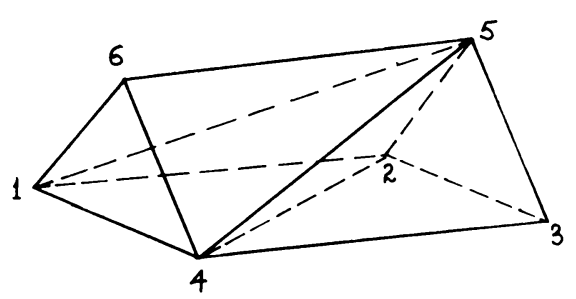

FigURE 4a

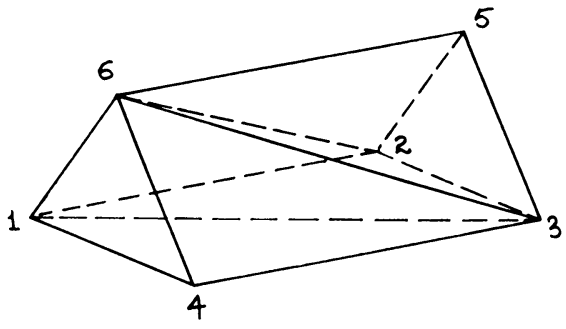

FIGURE 4b

Thus $T_{\theta}$ is a 6 vertex neighbourly triangulation of $S^{3}$. (Note that the vertices of Figure 4 are labelled so as to show that in fact $T_{\theta}$ is isomorphic to the cyclic triangulation $C_{3}^{6}$ of (4.1).) One checks that the two 2-simplices corresponding to $\{1,3,5\}$ and $\{2,4,6\}$ have not already been used. Taking the join of one of these triangles over the boundary of the other we can fill the hole in question.

By adding the simplices used in this patching and filling process to $K_{\sigma_{1}} \cup K_{\sigma_{2}}$ we thus obtain a triangulation of $\left(\sigma_{1}^{2} \cup \sigma_{2}^{2}\right) \times N^{2}$. We continue this process till all the 1 -simplices $\sigma^{1}$ of $L_{1}$ are exhausted. This gives us the required neighbourly triangulation $K$ of $M^{2} \times N^{2}$.

\section{Neighbourliness of 3-manifolds.}

(3.1) That all 3-manifolds admit combinatorial triangulations was proved by Moise [21]; the following refinement is due to Bing [2].

(3.1.1) If $M^{3}$ is a connected 3-manifold with nonempty boundary $\partial M^{3}$, then it admits a triangulation $K$ all of whose vertices lie on $\partial^{3}$.

(3.1.2) A simplicial complex $K$ will be called circular (or Hamiltonian) if all its vertices lie on a subcomplex $L$ which triangulates $S^{1}$.

EXAMPLE. Let $K$ be obtained by deriving all the 2-simplices of a triangulation $T$ of $S^{2}$ having 5 or more vertices. Then $K$ is not circular. 
(3.1.3) If $M^{3}$ is a connected 3-manifold with a nonempty connected boundary $\partial M^{3}$, then it admits a triangulation $K$ such that the induced triangulation $\partial K$ of $\partial M^{3}$ is circular and contains all the vertices of $K$.

To prove this theorem we start with a triangulation $K$ as in (3.1.1) and then improve it step by step till the additional requirement, i.e. the circularity of $\partial K$, is also satisfied.

Since $\partial K$ is connected we can find a closed edge path $\lambda$ in $\partial K$ which contains all the vertices of $K$. Let $e=\{i, j\}$ be an edge of $\partial K$ and let $\lambda(e)$ denote the number of occurrences of $e$ in the edge path. If $\lambda(e)>1$ we derive a 2-simplex $\sigma^{2}$ of $\partial K$ incident to $e$; in the ensuing triangulation $K^{\prime}$ of $M^{3}$ we replace $\lambda$ by an edge path $\lambda^{\prime}$ which is the same as $\lambda$ except that one of the occurrences of $e$ is replaced by the two edges $\{i, \hat{\boldsymbol{\sigma}}\}$ and $\{\hat{\boldsymbol{\sigma}}, j\}$. We note that $\lambda^{\prime}(e)=\lambda(e)-1$. So without loss of generality we can assume at the very outset that $\lambda(e)=0$ or 1 for all edges $e$ of $\partial K$.

Having chosen $K$ and $\lambda$ as above we let $L$ be the connected 1-dimensional subcomplex of $\partial K$ formed by all edges $e$ such that $\lambda(e)=1$. Note that each vertex of $L$ has an even valence, i.e., is incident to an even number of edges of $L$. Define

$$
\{L\}=\frac{1}{2}\left(\sum_{a \in \operatorname{vert}(L)}\left(\operatorname{val}_{L}(a)-2\right)\right) ;
$$

we note that $\{L\}$ is a nonnegative integer and is zero if and only if $|L|$ is homeomorphic to $S^{1}$.

If $\{L\}>0$ let $a$ be a vertex of $L$ with $\operatorname{val}_{L}(a) \geqslant 4$. Let $U=\mathrm{St}_{\partial K}(a)$; Figure 5a shows a picture of $L \cap U$. For any choice of adjacent edges $e_{1}, e_{2}$ of $L \cap U$ we replace $L$ by the graph $L^{\prime}=L_{12}$, which is the same outside $U$ and inside $U$ as shown in Figure $5 \mathrm{~b}$. We assert that with a proper choice of $e_{1}, e_{2}$ the graph $L_{12}$ is connected. Otherwise the component $L_{12}^{\prime}$ of $a$ is a proper subset of $L_{12}$; its complement $L_{12}^{\prime \prime}=L_{12}-L_{12}^{\prime}$ is also connected because any point of $L_{12}^{\prime \prime}$ not on the open $\operatorname{arc}(b c)$

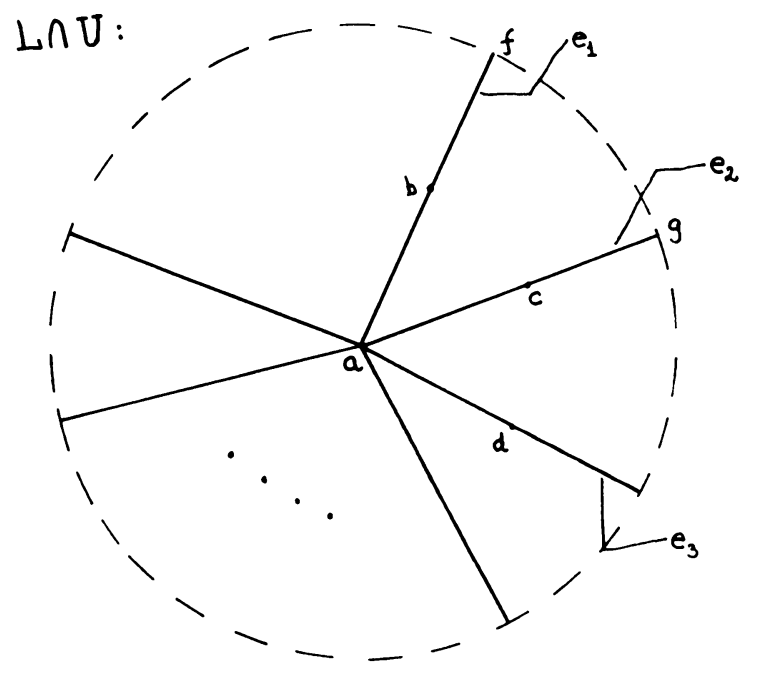

FIgURE 5a 


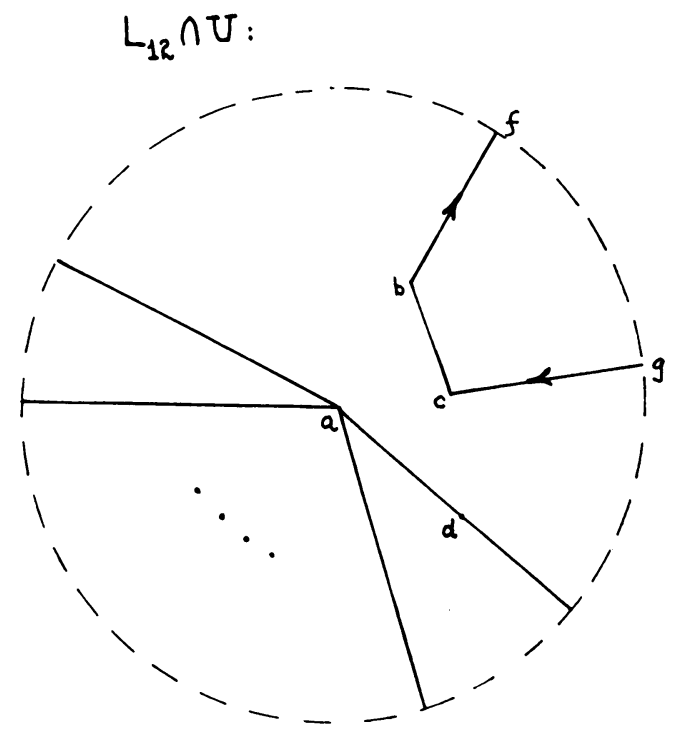

FIGURE 5b

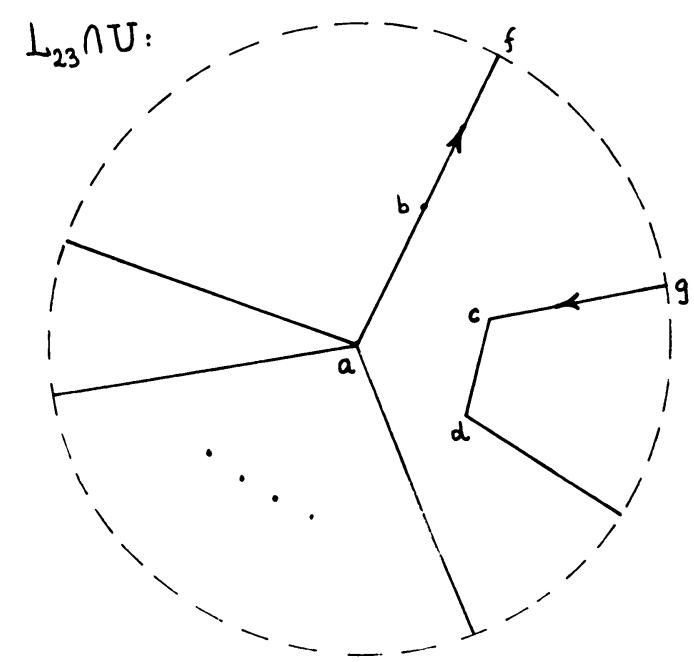

FIGURE 5c

must be a point of $L$ which can be joined in $L$ to $a$ via $b$ or $c$. Furthermore $L_{12}^{\prime \prime}-(b c)$ is connected because otherwise its component containing $b$ will furnish us with an example of a 1-dimensional complex in which exactly one vertex has an odd valence; an impossibility. The arrows in Figures $5 \mathrm{~b}$ and $5 \mathrm{c}$ show a path from $b$ to $c$ in $L_{12}^{\prime \prime}-(b c)$; this shows that if $L_{12}$ is not connected then $L_{23}$ will be connected and so proves the assertion.

The process $L \rightarrow L^{\prime}$ of the last paragraph can be accomplished simplicially. One enumerates the 1-simplices of the sector fag of $U=\mathrm{St}_{\partial K}(a)$ as $e_{1}=\sigma_{1}^{1}, \sigma_{2}^{1}, \ldots, \sigma_{k}^{1}=$ $e_{2}$ and then we derive them in succession at $b=\hat{\sigma}_{1}, \hat{\sigma}_{2}, \ldots, \hat{\sigma}_{k}=c$; this gives us a triangulation $K^{\prime}$ of $M^{3}$ in whose boundary $\partial K^{\prime}$ the arc [bc] is formed by the edges $\left\{\hat{\boldsymbol{\sigma}}_{1}, \hat{\boldsymbol{\sigma}}_{2}\right\},\left\{\hat{\boldsymbol{\sigma}}_{2}, \hat{\boldsymbol{\sigma}}_{3}\right\}, \ldots,\left\{\hat{\boldsymbol{\sigma}}_{k-1}, \hat{\boldsymbol{\sigma}}_{k}\right\}$. We note that $K^{\prime}, L^{\prime}$ have all the properties of $K, L$, respectively, and, in addition, $\left\{L^{\prime}\right\}=\{L\}-1$. This proves (3.1.3). 
(3.2) Let $\{a, b, c\}$ and $\{b, c, d\}$ be two 2-simplices lying on the boundary of a triangulated 3-manifold. If the 1-simplex $\{a, d\}$ does not belong to this triangulation, then one can obtain a new triangulation of the same 3-manifold by adding the tetrahedra $\{a, b, c, d\}$. One says that this new triangulation has been obtained from the old by patching $a$ to $d$. We note that this new triangulation has deficiency one less than the old; here, by deficiency of a simplicial complex having $x$ vertices, we mean the excess of $\left(\begin{array}{l}x \\ 2\end{array}\right)$ over the number of its edges.

(3.2.1) If $M^{3}$ is a closed connected 3-manifold, then there exists an integer $\nu$ such that for all integers $x \geqslant \nu, M^{3}$ admits a neighbourly triangulation $K^{x}$ with $x$ vertices.

The least integer $\nu$ having the above property will be denoted by $\nu_{M}$. We note that (3.2.1) is equivalent to the following theorem, since an extra vertex can be put in the missing 3 ball.

(3.2.2) If $M^{3}$ is a connected 3-manifold with boundary $S^{2}$, then there exists an integer $\nu$ such that for all integers $x \geqslant \nu, M^{3}$ admits a neighbourly triangulation $K^{x}$ with $x$ vertices, all lying on the boundary.

(3.2.3) We show first that an $M^{3}$ with $\partial M^{3}=S^{2}$ admits a neighbourly triangulation which satisfies all the properties of (3.1.3). To do this we pick any triangulation $K$ as in (3.1.3). If its deficiency is positive we will give a construction that enables us to reduce the deficiency by one without losing any of the properties of (3.1.3).

Let $L \subseteq \partial K$ be a circle such that all vertices of $K$ lie on $L$. Since $\partial K$ is a spherical triangulation one can find a 2-cell $T \subseteq \partial K$ such that $\partial T=L$. Let $x$ and $y$ be 2 distinct vertices of $K$ such that $\{x, y\}$ is not a 1 -simplex of $K$. These 2 vertices determine two arcs $L_{1}, L_{2} \subseteq L$ such that $\{x\} \cup\{y\}=\partial L_{1}=\partial L_{2}$. We cone the circles $L_{1} \cup\{x, y\}$ and $L_{2} \cup\{x, y\}$ over 2 new vertices $A$ and $B$, respectively, to get the cells $T_{1}$ and $T_{2}$; one has $\partial T_{1}=L_{1} \cup\{x, y\}$ and $\partial T_{2}=L_{2} \cup\{x, y\}$. Next we cone the spherical triangulation $T \cup T_{1} \cup T_{2}$ over yet another new vertex $X$ to get a triangulation $S$ of the 3-cell. It is clear that $K \cup S$ minus the tetrahedra $\left\{B, X, y, b_{q}\right\}$, where $b_{q}$ is the vertex of $L_{2}$ adjacent to $y$, is a new triangulation of (a manifold homeomorphic to) $M^{3}$. It has 3 more vertices than $K$, and all the vertices lie on a 2-cell of the boundary which is triangulated as in Figure 6a.

Note that the new vertex $X$ is joined (by a 1-simplex) to each of the other vertices. To ensure that $A$ is joined to all the other vertices we modify this triangulation of $M^{3}$ by patching $A$, in succession, to $B, b_{1}, b_{2}, \ldots, b_{q}$. The ensuing triangulation of $M^{3}$ has all its vertices on a 2-cell of the boundary which is triangulated as in Figure $6 \mathrm{~b}$. Finally to ensure that $B$ is joined to all other vertices we patch $B$, in succession, to $a_{1}, a_{2}, \ldots, a_{p}$. In so doing we pass from Figure $6 \mathrm{~b}$ to Figure $6 \mathrm{c}$. We note that this new triangulation of $M$ has deficiency one less than $K$ and has all its vertices on the boundary of the shaded 2-cell of Figure 6c. This proves the required assertion.

(3.2.4) To complete the proof of (3.2.2) we now show that if an $M^{3}$ with $\partial M^{3}=S^{2}$ admits a neighbourly triangulation with $n$ vertices satisfying the properties of (3.2.3) then it also admits one with $n+1$ vertices. Let the $n$ vertices of $K$ lie on a circle $L \subseteq \partial K$. We enumerate them in cyclic order as $v_{1}, v_{2}, \ldots, v_{n}$ and we choose a 


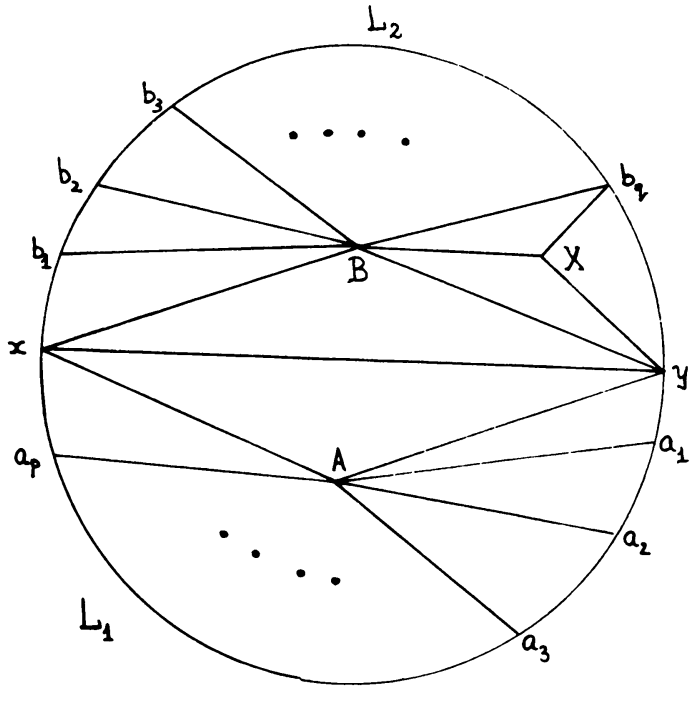

FIGURE 6a
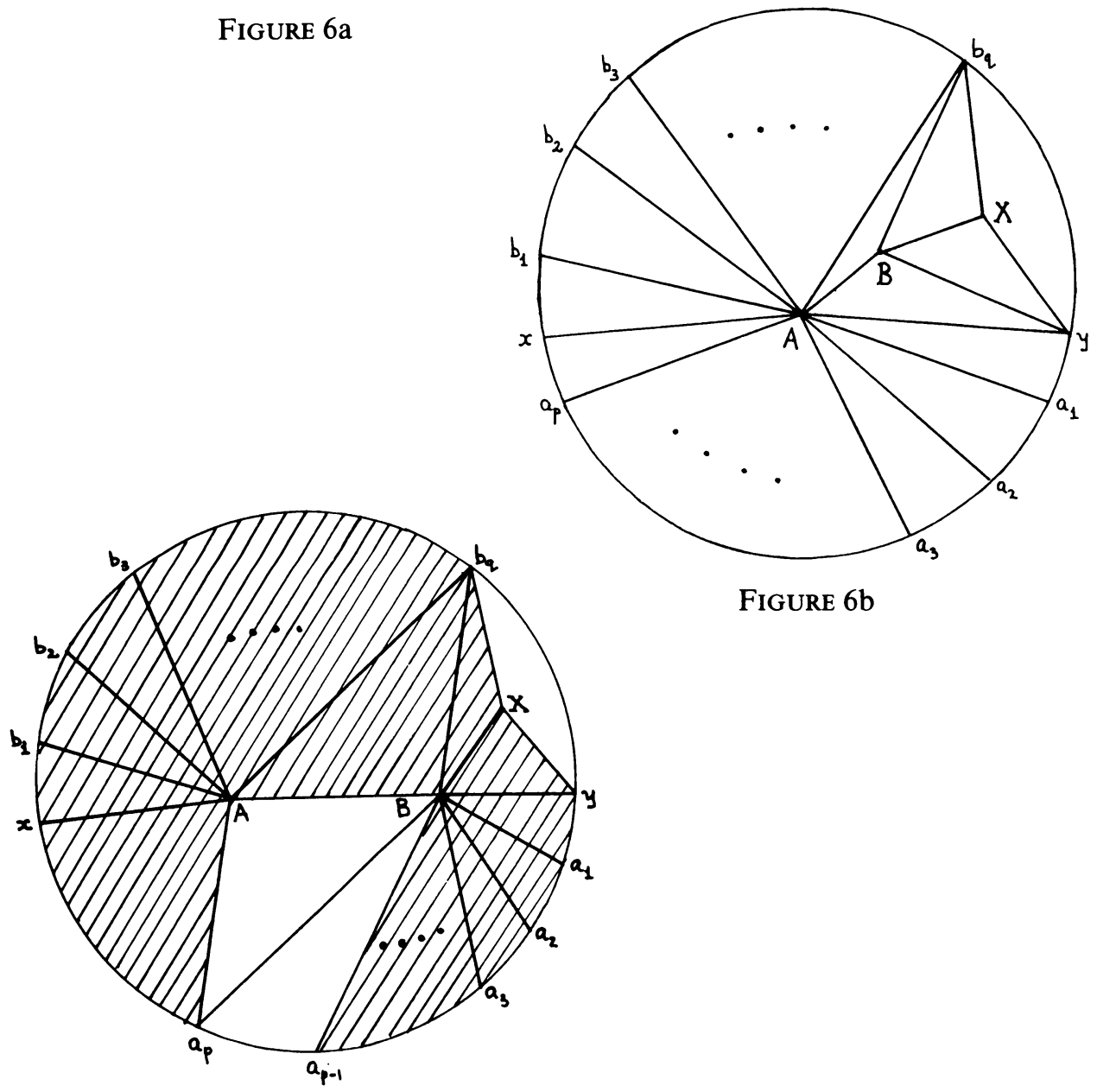

FigURE 6b

FIGURE 6c 
2-cell $T \subseteq \partial K$ such that $\partial T=L$. If $w$ is a new vertex we see that $K^{\prime}=K \cup(w \cdot T)$ is also a neighbourly triangulation of $M^{3}$ all of whose vertices lie on the circle $L^{\prime}=\left\{w, v_{1}\right\} \cup\left\{v_{1}, v_{2}\right\} \cup \cdots \cup\left\{v_{n-1}, v_{n}\right\} \cup\left\{v_{n}, w\right\}$ contained in $\partial K^{\prime}$.

(3.2.5) The conclusion of (3.2.2) holds for all connected 3-manifolds $M^{3}$ with $a$ nonempty connected boundary $\mathrm{\partial M}^{3}$.

One needs to strengthen (3.1.3) and show that all vertices lie on a circle $L$ whose imbedding in $\partial M^{3}$ is homotopically trivial. By examining the proof of (3.1.3) the reader will see that this is very easy to accomplish; one starts with a homotopically trivial closed edge path $\lambda$ and checks that the process $L \rightarrow L^{\prime}$ given there does not effect the homotopy type of the inclusion. The triviality of the imbedding $L \subseteq \partial K$ will ensure that we can choose the requisite 2-cells $T$ of (3.2.3) and (3.2.4).

\section{Order-orientable triangulations.}

(4.1) A set of integers is called contiguous if any integer lying between two members of this set is also in the set. (We can analogously speak of the contiguous subsets of any totally ordered set.) For each pair of integers $n, x, n \geqslant 0, x \geqslant n+2$, we define $C_{n}^{x}$ to be the simplicial complex generated by all $n$-simplices $\sigma \subseteq$ $\{1,2, \ldots, x\}$ having the property that any maximal contiguous subset of $\sigma$ which has odd cardinality contains 1 or $x$.

(4.1.1) For each integer $x \geqslant n+2$, the $n$-sphere $S^{n}, n \geqslant 0$, admits the [( $\left.\left.n-1\right) / 2\right]-$ neighbourly triangulation $C_{n}^{x}$.

These cyclic triangulations of the sphere were discovered by Carathéodory [4], but their combinatorial structure was understood only after their rediscovery by Gale [10] and Motzkin [22]. The definition of $C_{n}^{x}$ given above is based on "Gale's evenness condition" as given in McMullen and Shephard [20, p. 85].

Note that the asserted neighbourliness is a simple consequence of the definition of $C_{n}^{x}$. Furthermore it is clear that the unique triangulation of $S^{0}$ is (isomorphic to) $C_{0}^{x}$ for all $x \geqslant 2$ and that the triangulation of $S^{1}$ with $x$ vertices is $C_{1}^{x}$; again it is obvious that $C_{n}^{n+2}$ is the boundary complex of an $n+1$ simplex and so is a triangulation of $S^{n}$ for all $n$. Assume now that $C_{n}^{x}$ triangulates $S^{n}$; this implies that its subcomplex $K=C_{n}^{x}-\operatorname{St}(x)$ triangulates the $n$-disk. We define $L$ (resp. $L^{\prime}$ ) to be the subcomplex of $\partial K\left(=C_{n-1}^{x-1}\right)$ generated by all $n-1$ simplices $\sigma$ such that the maximal contiguous subset of $x . \sigma$ containing $x$ has odd (resp. even) cardinality. It is easy to verify that besides $L \cup L^{\prime}=\partial K$ one also has $L \cap L^{\prime}=\partial L=\partial L^{\prime}=C_{n-2}^{x-1} \subseteq \partial K$. Hence $L$ and $L^{\prime}$ are the $n-1$ disks formed by taking the closure of the two components into which the $n-1$ sphere $\partial K$ is separated by the $n-2$ sphere $C_{n-2}^{x-1} \subseteq \partial K$. It follows that $K^{\prime}=K \cup(x+1 . L)$ is a triangulation of an $n$-disk and so $K^{\prime} \cup\left(x . \partial K^{\prime}\right)$ is a triangulation of the $n$-sphere. One can check that this is nothing but $C_{n}^{x+1}$. This proves (4.1.1).

Besides these cyclic triangulations $S^{n}$ admits many other [( $\left.\left.n-1\right) / 2\right]$-neighbourly triangulations (for $n \geqslant 2$ ); some of these can be constructed by using the following lemma. This result may also turn out to be helpful in proving some generalisations of the results of $\S 3$. 
(4.1.2) Suppose that the manifold $M^{n}$, with nonempty boundary, admits a $d$ neighbourly triangulation $K$ having $\nu$ vertices. Suppose further that there exists a sequence of disks $D_{1}, D_{2}, \ldots, D_{d}$ of dimensions $n-1, n-2, \ldots, n-d$, respectively, such that (a) $D_{1} \subseteq \partial K$ and $D_{i+1} \subseteq \partial D_{i}$ for $1 \leqslant i \leqslant d-1$ and (b) $\partial D_{i}, 1 \leqslant i \leqslant d$, contains all simplices of $K$ with dimensions $\leqslant d-i$. Then for each integer $x \geqslant \nu, M^{n}$ admits a d-neighbourly triangulation with $x$ vertices.

Let $w$ be a new vertex and replace $K$ by $K^{\prime}=K \cup\left(w . D_{1}\right)$. Then $K^{\prime}$ is a $d$-neighbourly triangulation of $M^{n}$ with $\nu+1$ vertices. Choose any $n-d-1$ dimensional simplex $\sigma$ of $\partial D_{d}$; then the disks $D_{1}^{\prime}=w . \partial D_{1}-w \cdot D_{2}, D_{2}^{\prime}=w . \partial D_{2}-$ $w . D_{3}, \ldots, D_{d}^{\prime}=w . \partial D_{d}-w . \sigma$ have the properties (a) and (b) vis-à-vis $K^{\prime}$. This proves (4.1.2).

(4.2) Let $M^{n}$ be an orientable manifold; so $H_{n}\left(M^{n}, \partial M^{n} ; \mathbf{Z}\right)$ is isomorphic to $\mathbf{Z}$. Let $K$ be any triangulation of $M^{n}$. A total ordering of the vertices of $K$ associates to each simplex $\sigma$ of $K$ an oriented simplex [ $\sigma$ ]. We say that $K$ is order-orientable if a total ordering exists such that the sum of the oriented $n$-simplices is a cycle of $K \bmod \partial K$ and so determines a generator of $H_{n}(K, \partial K ; \mathbf{Z}) \cong H_{n}\left(M^{n}, \partial M^{n} ; \mathbf{Z}\right)$. We will say that this total ordering orients $K$.

(4.2.1) If $K$ is an order-orientable triangulation of $M^{n}$, then any simplex of $K$ having dimension $\leqslant[(n-2) / 2]$ lies on $\partial M^{n}$.

This is an immediate consequence of the following result.

(4.2.2) If the vertices of $K$ are labelled as $1,2, \ldots, x$ in accordance with a total ordering which orients $K$, then (int $K$ ) $\cap C_{n-1}^{x}=\varnothing$.

Let $\sigma^{k}$ be a simplex of $C_{n-1}^{x} \cap K$; then it has at most $n-1-k$ maximal contiguous subsets of odd cardinality containing neither 1 nor $x$. This assertion remains true if we replace 'contiguous' by ' $\sigma$-contiguous'-i.e. contiguous when considered as a subset of the smaller totally ordered set vert $\left(\overline{\mathrm{St}}_{K} \sigma\right)$ - and ' 1 nor $x$ ' by ' $1_{\sigma}$ nor $x_{\sigma}$ ' where $1_{\sigma}$ (resp. $\left.x_{\sigma}\right)$ is the smallest (resp. biggest) vertex in $\operatorname{vert}\left(\overline{\operatorname{St}}_{K} \sigma\right)$. We can now choose a simplex $\phi^{k+1} \in \mathrm{St}_{K} \sigma$ such that it has at most $n-1-k-1$ maximal $\sigma$-contiguous subsets of odd cardinality containing neither $1_{\sigma}$ nor $x_{\sigma}$. Again we can replace ' $\sigma$-contiguous' by ' $\phi$-contiguous' and ' $1_{\sigma}$ nor $x_{\sigma}$ ' by ' $1_{\phi}$ nor $x_{\phi}$ '. Continuing in this way we choose an $n-1$ simplex $\theta^{n-1} \in \mathrm{St}_{K} \sigma$ which has no maximal $\boldsymbol{\theta}$-contiguous subsets of odd cardinality containing neither $1_{\theta}$ nor $x_{\boldsymbol{\theta}}$. Using the well-known formula $\partial\left[a_{0}, a_{1}, \ldots, a_{n}\right]=\sum_{i=0}^{n}(-1)^{i}\left[a_{0}, a_{1}, \ldots, \hat{a}_{i}, \ldots, a_{n}\right]$ it follows that the sum of the oriented $n$-simplices of $K$ has $\pm r_{\theta}[\theta]$ in its boundary; here the sign depends on whether the maximal $\theta$-contiguous subset of $\theta$ containing $1_{\theta}$ is of even or odd cardinality and $r_{\theta}=1$ or 2 depending on whether $\theta \in \partial K$ or int $K$. Since our ordering orients $K$ we must have $\theta^{n-1} \in \partial K$ and so its face $\sigma^{k}$ also lies in $\partial K$.

We note the following corollary. 
(4.2.3) If a manifold $M^{n}, n \geqslant 1$, admits an order-orientable triangulation, then it has a nonempty boundary $\partial M^{n}$ and the inclusion map $\partial M^{n} \rightarrow M^{n}$ induces an isomorphism $H_{i}\left(\partial M^{n}\right) \rightarrow H_{i}\left(M^{n}\right)$ for $i<[(n-2) / 2]$.

(4.2.4) The 2-disk is the only 2-dimensional manifold which admits an order-orientable triangulation $K$; for such $a K$ the conclusion of (4.2.2) can be strengthened to $\partial K=C_{1}^{x}$.

We leave the proof of the second part to the reader and show how it implies the first. Let $L$ be triangulation of a 2-manifold other than the 2-disk. Then $L$ can be obtained from a triangulation $K$ of the 2-disk, having the same number of triangles as $L$, by identifying some pairs of edges on $\partial K$. If $\pi: \operatorname{vert}(K) \rightarrow \operatorname{vert}(L)$ is the map defined by these identifications we can obviously lift any total ordering on $\operatorname{vert}(L)$ to a total ordering on vert $(K)$ under which (i) $\pi$ is order preserving and (ii) each fiber of $\pi$ is a contiguous subset of $\operatorname{vert}(K)$. If the ordering of $\operatorname{vert}(L)$ orients $L$ then such a lifting would orient $K$. Label the vertices of $K$ as $1,2, \ldots, x$ in accordance with this total ordering: by the second part of (4.2.4) we must have $\partial K=C_{1}^{x}$. Hence if $a$ and $b$ are 2 vertices of $\partial K$ such that $\pi(a)=\pi(b)$ it follows, using (ii), that every vertex $v$ of $\partial K$ lying on one of the two arcs joining $a$ to $b$ is such that $\pi(v)=\pi(a)$; in particular there will be one such vertex $v$ such that the 1-simplex $\{a, v\}$ lies in $\partial K$. This is not possible; so no total ordering on vert $(L)$ can orient $L$.

(4.2.5) EXAMPLES. (a) For each pair of integers $x, n, n \geqslant 1, x \geqslant n+1$, we denote by $D_{n}^{x}$ the $[(n-1) / 2]$-neighbourly triangulation of the $n$-disk obtained by deleting the star of $x+1$ from $C_{n}^{x+1}$. Note that $\partial D_{n}^{x}=C_{n-1}^{x}$. It is easily verified that the natural order of the integers orients $D_{n}^{x}$.

(b) The proof of (4.3.2) will show how one can construct numerous order-orientable triangulations $K$ of the $n$-disk, $n \geqslant 1$, for which the conclusion of (4.2.2) can be strengthened to $\partial K=C_{n-1}^{x}$. However there exist order-orientable triangulations of the 3-disk for which $\partial K$ is not isomorphic to a cyclic triangulation. If $x$ is an integer $\geqslant 5$, then the triangulation $K$ of the 3-disk which is the join of the 1-simplex $\{2, x-1\}$ and the triangulation $\{1, x\},\{x, 3\},\{3,4\}, \ldots,\{x-3, x-2\},\{x-2,1\}$ of the circle is oriented by the natural order of the integers. However for $x \geqslant 6, \partial K$ is not isomorphic to $C_{2}^{x}: C_{2}^{x}$ has exactly 2 vertices of valence 3 while $\partial K$ has none.

(c) Any three-dimensional handlebody admits an order-orientable triangulation. (By a handlebody we mean a 'pretzel with $g$ holes'; $g$ is called the genus of the handlebody.) In fact we will inductively construct, for each integer $g \geqslant 0$, a simplicial complex $T_{g}$ such that (i) $T_{g}$ is a triangulation of the handlebody of genus $g$, (ii) $\operatorname{vert}\left(T_{g}\right)=\{1,2,3, \ldots, 5 g+6\}$, (iii) the triangles $\{1,2,3\}$ and $\{5 g+4,5 g+5,5 g+$ $6\}$ lie on $\partial T_{g}$, and (iv) the natural order of the integers orients $T_{g}$. We define $T_{0}$ to be the triangulation of the 3-disk shown in Figure 7a; obviously it satisfies the required conditions for $g=0$. For each $g \geqslant 0$, the simplicial complex $T_{g}$ gives rise to an isomorphic simplicial complex $\tilde{T}_{g}$ if we replace the vertices $1,2,3, \ldots, 5 g+6$ by $1,2,4,8,9, \ldots, 5 g+10$, respectively. By (iii) the triangles $\{1,2,4\}$ and $\{5 g+8,5 g$ $+9,5 g+10\}$ lie on $\partial \tilde{T}_{g}$. We obtain $T_{g+1}$ from $\tilde{T}_{g}$ by attaching the handle $H_{g}$ of Figure $7 \mathrm{~b}$ to these 2 triangles, i.e. we set $T_{g+1}=\tilde{T}_{g} \cup H_{g}$. 


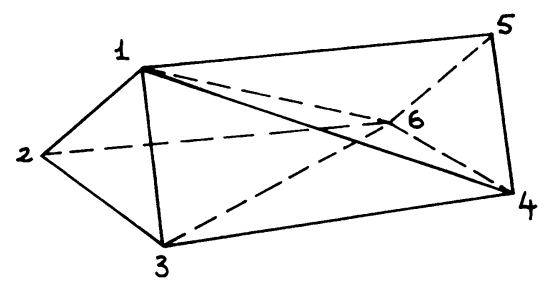

Figure 7a

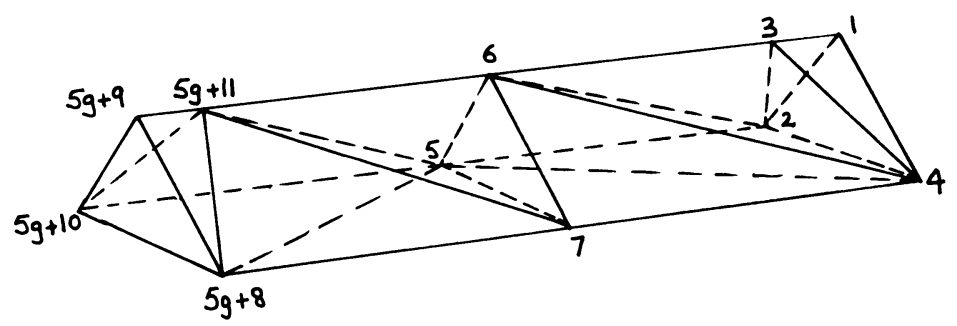

FIGURE $7 b$

$T_{g+1}$ obviously satisfies (i), (ii) and (iii). To verify (iv) we note, from Figure 7b, that the natural order of the integers orients $H_{g}$ and it induces positive (resp. negative) orientation on the triangle $\{1,2,4\}$ (resp. $\{5 g+8,5 g+9,5 g+10\}$ ); on the other hand, by the inductive hypothesis, the same order orients $\tilde{T}_{g}$ and induces negative (resp. positive) orientation on the triangle $\{1,2,4\}$ (resp. $\{5 g+8,5 g+$ $9,5 g+10\})$.

(d) The reader can check that a triangulation $K$ of the 2-disk with all vertices on $\partial K$ is necessarily order-orientable. The analogous statement for 3-manifolds is false. Consider, e.g., the seven tetrahedra triangulation $Q$ of the solid torus shown in Figure $8 \mathrm{a}$ (where the two triangles on the left and right are identified as per the labelling). Figure $8 \mathrm{~b}$ shows the boundary triangulation $\partial Q$ of the 2-torus.

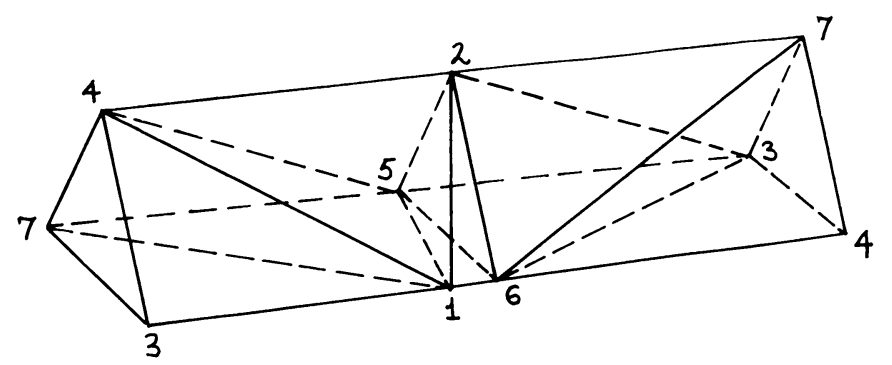

FIGURE 8a

The triangulation $Q$ of the solid torus is not order-orientable. The seven tetrahedra of $Q$ are $\{1,2,4,5\},\{1,2,6,7\},\{1,3,4,7\},\{1,4,5,7\},\{2,3,5,6\},\{2,3,6,7\}$ and $\{3,4,6,7\}$. Each cyclic permutation of 1234567 induces a simplicial isomorphism $Q \rightarrow Q$. So if a permutation of 1234567 were to orient $Q$ there would also be one such which has 4 as its first entry. Then the induced total order on Lk 4 would orient this 2 -disk. We note that $\partial(\operatorname{Lk} 4)$ is precisely the bounding circle of the shaded part 
of Figure $8 \mathrm{~b}$. Using (4.2.4) we are thus reduced to ruling out just 12 possibilities, viz. $4136752,4367521, \ldots$ and $4125763,4257631, \ldots$ One can check that none of these orients $Q$.

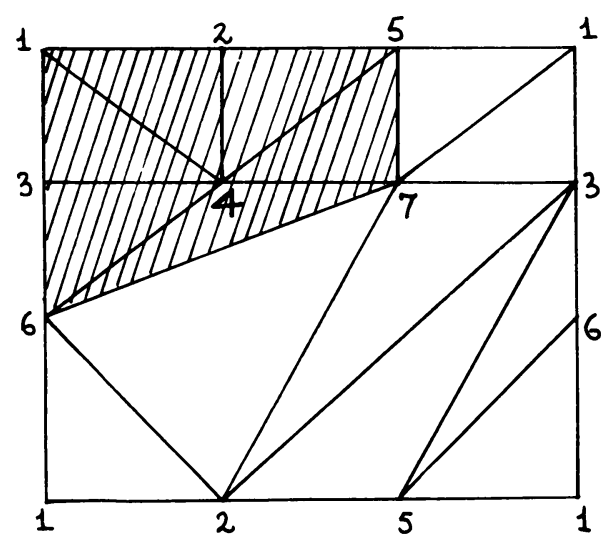

FIGURE 8b

Some more remarks regarding $Q$ may be of interest. The 2-torus (resp. solid torus) has no triangulation with less than 7 vertices; $\partial Q$ (resp. $Q$ ) is, up to simplicial isomorphism, the unique triangulation with 7 vertices. $Q$ is a subcomplex of the cyclic triangulation $C_{3}^{7}$; the seven tetrahedra of $C_{3}^{7}$ which are not in $Q$ determine another solid torus $Q^{\prime}(\cong Q)$ : the minimal triangulation of the 2-torus splits up the seven vertex cyclic triangulation of the 3-sphere into two solid tori each equipped with a minimal triangulation. The automorphism group of $C_{3}^{7}$ is the dihedral group formed by cyclic permutations and reversals of 1234567 ; each of these automorphisms preserves the two solid tori. This group is a normal subgroup of the group of automorphisms of $\partial Q=\partial Q^{\prime}$; the quotient being a cyclic group of order 3 . Correspondingly the minimal triangulation of the 2-torus has 3 distinct extensions $Q, Q^{\prime}$, $Q^{\prime \prime}\left(Q \cong Q^{\prime} \cong Q^{\prime \prime}\right)$ which are 7 vertex triangulations of the solid torus; and the union of any 2 of these extensions is a triangulation of the 3-sphere isomorphic to $C_{3}^{7}$.

It is interesting to note that a geometric construction of the minimal torus $Q$ was first discovered by Möbius [36, pp. 552-553] and rediscovered by Császár [34].

(4.3) Throughout this section $A^{n}, n \geqslant 1$, will denote an $n$-dimensional real affine space and $X$ will be a finite subset of $A^{n}$ containing $x \geqslant n+1$ points which are in general position, i.e. no proper affine subspace of $A^{n}$ contains $n+1$ points of $X$. We denote by $K_{X}$ the triangulation of the $n-1$ sphere determined by the boundary facets of $\operatorname{conv}(X)$, the convex hull of $X$. We need the following lemma.

(4.3.1) If $\sigma^{k} \subseteq X \subseteq A^{n}, 0 \leqslant k \leqslant n$, is a $k$-simplex, then there exists a simplicial complex $K_{\sigma}$ such that $\operatorname{vert}\left(K_{\sigma}\right) \subseteq X, \sigma^{k} \in K_{\sigma}, K_{X} \subseteq K_{\sigma}$ and $\left|K_{\sigma}\right|=\operatorname{conv}(X)$. 
Choose an origin and a basis for $A^{n}$ and thereby identify $A^{n}$ with $\mathbf{R}^{n}$. Define $\tilde{X} \subseteq \mathbf{R}^{n+1}=\mathbf{R}^{n} \times \mathbf{R}$ by $\tilde{X}=\left\{\left(v, i_{v}\right): v \in X, i_{v}=0\right.$ if $v \notin \sigma, i_{v}=1$ if $\left.v \in \sigma\right\}$. We can assume $x>n+1$; so $\operatorname{conv}(X)$ is an $n+1$ disk and the boundary facets which are farther away from the hyperplane $\mathbf{R}^{n} \times\{0\}$ determine a polyhedral subdivision of an $n$-disk. Using the canonical linear surjection $\mathbf{R}^{n} \times \mathbf{R} \rightarrow \mathbf{R}^{n}$ this images to a polyhedral subdivision $\tilde{K}_{\sigma}$ of $\operatorname{conv}(X)$ having the desired properties. It is clear that one can subdivide $\tilde{K}_{\sigma}$ further, without increasing vertices, to get a simplicial complex $K_{\sigma}$ with the same properties.

As an application of (4.2.2) we now give a new proof of the following result which is presumably well known.

(4.3.2) If the elements of $X \subseteq A^{n}$ are labelled as $1,2, \ldots, x$ in accordance with a total ordering under which any $n+1$ points of $X$ determine the same orientation of $A^{n}$, then $K_{X}=C_{n-1}^{x}$.

Let $\sigma^{k} \in C_{n-1}^{x}$; since $\sigma^{k} \subseteq X$ we can find a simplicial complex as in (4.3.1). The given hypothesis on the total ordering implies that $K_{\sigma}$ is an order-orientable triangulation of the $n$-disk conv $(X)$. So, by (4.2.2), $\sigma^{k}$ cannot meet the interior of $\operatorname{conv}(X)$. Hence $\sigma^{k} \in K_{X}$. Since the spherical triangulation $C_{n-1}^{x}$ cannot be a proper subcomplex of the spherical triangulation $K_{X}$ we must have $K_{X}=C_{n-1}^{x}$.

We have thus come back to the point from which Carathéodory [4] et al. discovered the cyclic triangulations. For example Gale [11] defines it to be $K_{X}$, where $X \subseteq \mathbf{R}^{n}$ consists of $x$ distinct points on the moment curve $t \mapsto\left(t, t^{2}, \ldots, t^{n}\right)$; clearly such an $X$ satisfies the hypothesis of (4.3.2).

(4.3.3) $X \subseteq A^{n}$ will be called a monotone set if it admits a total ordering as in (4.3.2). The elements of $X$ arranged according to such an ordering form an associated monotone sequence. This terminology is due to Hjelmslev [14]. We will now study the following problem (which is the affine version of the one) raised by Uhrin [29] and H. Gupta: Can the monotonicity of a set $X \subseteq A^{n}$ be characterised by means of some geometric properties?

(4.3.4) Let us first study the lower-dimensional cases. We point out that for $n \leqslant 2$ the solution is contained in Hjelmslev [14].

Case $n=1$. This is trivial: any $X \subseteq A^{1}$ is monotone and each of the two orientations of $A^{1}$ determines uniquely an associated monotone sequence.

Case $n=2$. By (4.3.2) we see that $X \subseteq A^{2}$ is monotone only if each point of $X$ lies on the boundary of the 2-disk $\operatorname{conv}(X)$. Conversely this geometric criteria ensures the monotonicity of $X$ : take any point of $X$ as the least point and arrange the other points in sequence as one traces out the circle $K_{X}$. Thus each orientation of $A^{2}$ determines precisely $x$ associated monotone sequences.

Case $n=3$. By (4.3.2) we see that $K_{X}$ must be isomorphic to $C_{2}^{x}$. One can describe $C_{2}^{x}$ as the boundary of the triangulation of the 3-disk obtained by taking the join of a l-simplex $\left\{v_{1}, v_{2}\right\}$ with the triangulation $I_{k}$ of the interval having $k=x-2$ vertices. (Figure 9 indicates this for $x=7$.) It follows that for $x \geqslant 6, \operatorname{Aut}\left(C_{2}^{x}\right)$ is the four group whose elements are the permutations $12 \ldots x, x 23 \ldots x-11,1 x-1$ $x-2 \ldots 32 x$ and $x x-1 x-2 \ldots 321$. 


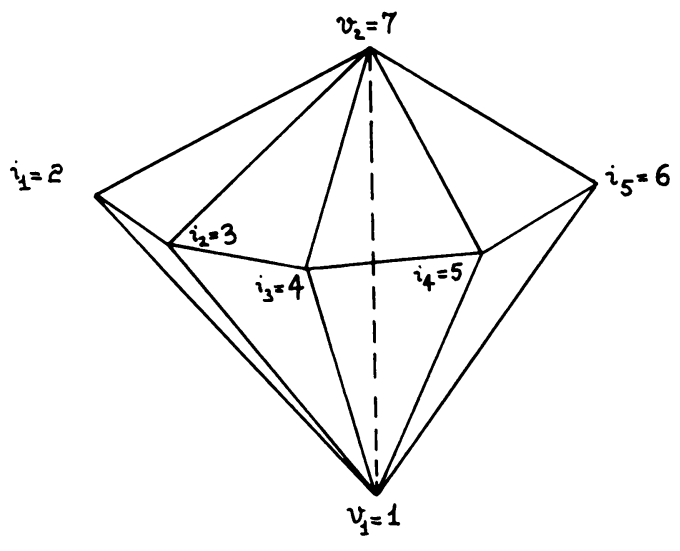

Figure 9

There exists a nonmonotone $X \subseteq A^{3}$ with $x=7$ such that $K_{X} \cong C_{2}^{x}$. Let $X=$ $\{1,2,3,4,5,6,7\} \subseteq A^{3}$ be as in Figure 9 and such that 2 and 6 lie on the same side of the plane of $\{3,4,5\}$. If $X$ were monotone, then-by (4.3.2) and the above remark regarding $\operatorname{Aut}\left(C_{2}^{7}\right)$-it follows that either 1234567 or 1654327 must be an associated monotone sequence; this cannot happen because 2 and 6 lie on the same side of the plane of $\{3,4,5\}$.

If $x \geqslant 6$ the necesary condition $K_{X} \cong \partial\left(v_{1} v_{2} . I_{x-2}\right)$ narrows down the search for monotone sequences to just 2 possibilities, viz. $v_{1} i_{1} i_{2} \cdots i_{x-2} v_{2}$ and $v_{1} i_{x-2} i_{x-3} \cdots i_{1} v_{2}$. This follows from (4.3.2) and the remark regarding $\operatorname{Aut}\left(C_{2}^{x}\right), x \geqslant 6$. Note that at most one of those possibilities holds; of course in that case its reversal is also a monotone sequence. Thus if $X \subseteq A^{3}, x \geqslant 6$, is monotone then one of the orientations of $A^{3}$ determines 2 associated monotone sequences while the other determines none.

To give a similar necessary condition for all $n \geqslant 4$ we will isolate those aspects of the simplicial structure of $C_{n-1}^{x}$ which do not depend on the natural order of the integers.

(4.3.5) If $X \subseteq A^{n}, x \geqslant n+3$, is a monotone set the simplicial complex $K_{X}$ has $x$ vertices and is $[(n-2) / 2]-n e i g h b o u r l y . ~ F u r t h e r ~ i f ~ n$ is odd (resp. even) then the link of precisely two vertices (resp. of all vertices) $v$ has $x-1$ vertices and is $[(n-3) / 2]-$ neighbourly and, for each such vertex $v$, one can define in a canonical way a monotone set $X_{v} \subseteq A^{n-1}$ with $x_{v}=x-1$ and an isomorphism $\pi_{v}: \operatorname{Lk}(v) \stackrel{\cong}{\rightarrow} K_{X_{v}}$. Conversely these necessary conditions ensure that the search for a monotone sequence can be restricted to those sequences which (i) start with a fixed vertex $v$ of $K_{X}$ whose link has $x-1$ vertices and is $[(n-3) / 2]-n e i g h b o u r l y$, (ii) are such that the sequence induced under $\pi_{v}$ on $X_{v}$ is monotone, and (iii) end with another vertex of the same type as $v$. (The definition of $X_{v}$ and of $\pi_{v}$ will be given in the proof below.)

If $X \subseteq A^{n}$ is monotone we have $K_{X} \cong C_{n-1}^{x}$ by (4.3.2) and so à fortiori $K_{X}$ has $x$ vertices and is [(n-2)/2]-neighbourly. If $n$ is even the link of every vertex of $K_{X}$ is isomorphic to $C_{n-2}^{x-1}$ and so has $x-1$ vertices and is $[(n-3) / 2]$-neighbourly. To see 
this note, since $n$ is even, that a cyclic permutation of a monotone sequence is also a monotone sequence. Since link of $x$ in $C_{n-1}^{x}$ is $C_{n-2}^{x-1}$ the assertion follows. For any $n$, the reversal of a monotone sequence is also a monotone sequence; so by the same argument there exist at least two vertices of $K_{X}$ whose link has $x-1$ vertices and is [(n-3)/2]-neighbourly. If $n$ is odd, let $k$ denote the integer $(n-3) / 2$; so $k+2=$ $(n-1)-k$. For each integer $i$ such that $1<i<x$ we can choose, since $x \geqslant n+3$, a simplex $\sigma^{k+1}$ of the type $\{(1<) i-2 p, i-2 p+2, \ldots, i-2, i, i+2, \ldots, i+2 m$ $-2, i+2 m(<x)\}$. Since $\sigma^{k+1} \subseteq\{1,2, \ldots, x\}$ has $k+2>(n-1)-k-1$ maximal contiguous subsets of odd cardinality containing neither 1 nor $x$ it follows that $\sigma^{k+1} \notin C_{n-1}^{x}$, and so the simplex $\theta^{k}$ defined by $\sigma^{k+1}=i . \theta^{k}$ does not lie in the link of $i$. Thus, if $n$ is odd, there are precisely 2 vertices of $K_{X}$ whose link has $x-1$ vertices and is $[(n-3) / 2]$-neighbourly.

For any $v \in X$ one can choose an affine hyperplane $A^{n-1} \subseteq A^{n}$ which separates $v$ from the other points of $X$; this is possible since all the points of $x$ are the vertices of the convex polytope $\operatorname{conv}(X)$. We note that a line joining $v$ to any other point of $X$ thus meets $A^{n-1}$ in a unique point; we define $X_{v} \subseteq A^{n-1}$ to be the set of all such points. Since $X \subseteq A^{n}$ is in general position $X_{v} \subseteq A^{n-1}$ contains $x-1$ points and is in general position. It is clear that the natural map $\pi_{v}: X-\{v\} \rightarrow X_{v}$ induces an isomorphism $\pi_{v}: \operatorname{Lk}(v) \stackrel{\cong}{\rightarrow} K_{X_{v}}$. If $\operatorname{Lk}(v)$ has $x-1$ vertices and is $[(n-3) / 2]-$ neighbourly, then we see from the last paragraph that there must be a monotone sequence associated to $X \subseteq A^{n}$ which starts with $v$; so $X_{v} \subseteq A^{n-1}$ is monotone. The converse is also obvious from the last paragraph. So (4.3.5) follows.

If $X \subseteq A^{n}, x \geqslant n+3$, is a monotone set and $n$ is odd (resp. even) then there are exactly 2 (resp. $2 x$ ) associated monotone sequences.

We will prove this by induction on $n$. The fact that there are at least so many monotone sequences is obvious because if $n$ is odd (resp. even), then one can reverse (resp. reverse and cyclically permute) any monotone sequence to get a new one. We choose a vertex $v$ of $K_{X}$ whose link has $x-1$ vertices and is [( $\left.n-3\right) / 2$ ]-neighbourly. If $n$ is even, $x$ such choices of $v$ are possible, and there are at most two monotone sequences starting with $v$, viz. those which induce on $X_{v} \subseteq A^{n-1}$ its two monotone sequences. If $n$ is odd, two such vertices $v_{1}$ and $v_{2}$ exist. Any monotone sequence starting with $v_{1}$ must induce on $X_{v_{1}}$ a monotone sequence ending with the vertex corresponding to $v_{2}$; thus there are two such possibilities, say $v_{1} a_{1} a_{2} \cdots a_{x-2} v_{2}$ and $v_{1} a_{x-2} a_{x-3} \cdots a_{1} v_{2}$. But one of them must be ruled out because if $n=1 \bmod 4$ (resp. 3 mod 4) then the two sequences assign the same (resp. different) orientation to the simplex $\left\{v_{1}, a_{1}, a_{2}, \ldots, a_{n}\right\}$ and different (resp. same) orientation to the simplex $\left\{a_{1}, a_{2}, \ldots, a_{n+1}\right\}$. Thus there are no more monotone sequences. If $n=0$ or 3 mod 4 all these monotone sequences determine only one of the two orientations: if $n=1$ or $2 \bmod 4$ the reversal of each monotone sequence determines the opposite orientation. This follows from the fact that one does not change the orientation of an $n$-simplex by reversing its vertices iff $n=0$ or $3 \bmod 4$. We note from the preceding that there are just 2 sequences satisfying (i), (ii) and (iii) of (4.3.5); so (4.3.5) can be considered as a reasonable generalisation of the case $n=3$ discussed before. 
(4.3.6) To formulate a linear version of the above, one introduces the notion of a monotone set of half rays. This is a finite set of half rays in the $n$-dimensional vector space $V^{n}$, which admits a total ordering under which any $n$ of the half rays determine the same orientation of $V$. Analogously one speaks of a monotone sequence of half rays. This terminology is due to Hjelmslev [14].

The n-dimensional linear version of problem (4.3.3) is equivalent to the $n-1$ dimensional problem (4.3.3). To see this note that we can pick one nonzero point on each half ray in such a way that these points together with the origin form a set $X \subseteq V^{n}$ which is in general position. We want to find a geometric characterisation of the requirement that $X$ admit a total ordering, with the origin as first point, under which any $n+1$ points of $X$ determine the same orientation of $V^{n}$. In the notation of (4.3.5), this is equivalent to characterising the monotonicity of $X_{0} \subseteq A^{n-1}$.

\section{Chromatic numbers.}

(5.1) We fix an infinite set $C$ whose objects will be called 'colours'; any function taking its values in $C$ is called a 'colouring'. If $i$ is an integer $\geqslant 0$ and $K$ (resp. $X$ ) is a simplicial complex (resp. triangulable space), then its ith chromatic number $\operatorname{ch}_{i}(K)$ (resp. $\left.\operatorname{ch}_{i}(X)\right)$ is defined to be the smallest positive integer such that the $i$-simplices of $K$ can be coloured in such a way that no $i+1$ simplex of $K$ has all its faces of the same colour (resp. $=\sup _{K} \operatorname{ch}_{i}(K)$, as $K$ runs over all the triangulations of $X$ ). This definition was given in [25].

The presence of neighbourly triangulations is often helpful in getting good lower bounds for these chromatic numbers. For example, if $M^{2}$ is a closed neighbourly 2-manifold then $\operatorname{ch}_{0}\left(M^{2}\right) \geqslant x_{M}$ where $x_{M}$ is the unique integer $\geqslant 4$ which satisfies $\operatorname{dim}\left(H_{1}\left(M ; \mathbf{Z}_{2}\right)\right)=(x-4)(x-3) / 6$; this follows from Theorem (2.1.1) of Ringel et al. Again, Theorem (3.2.1) ensures that $\operatorname{ch}_{0}\left(M^{3}\right)=\infty$. We will prove below, in (5.1.3), a more general infiniteness result by following a much simpler argument.

(5.1.1) If $i$ is an integer $\geqslant 0$ and $K(n)$ a simplicial complex consisting of all the faces of an $n$-simplex, then $\lim _{n \rightarrow \infty} \operatorname{ch}_{i}(K(n))=\infty$.

To prove this we need to formulate a lemma.

Definition of $o_{i}(K)$. If $i$ is an integer $\geqslant 0$ and $K$ any simplicial complex we set $o_{i}(K)=\inf o_{i}(f)$ where $f$ runs over all the colourings of the $i$-simplices of $K$ and $o_{i}(f)=\Sigma_{\alpha \in \operatorname{Im} f} f_{\alpha}, f_{\alpha}$ being the largest integer such that $f^{-1}(\alpha)$ contains all the $i$-simplices of a $K\left(f_{\alpha}-1\right)$. We note that

$$
\operatorname{ch}_{i}(K)=\inf \left\{o_{i}(f) /(i+1) \mid f \text { s.t. } f_{\alpha}=i+1 \forall \alpha \in \operatorname{Im} f\right\} ;
$$

so $\operatorname{ch}_{i}(K) \geqslant o_{i}(K) /(i+1)$ and (5.1.1) is a consequence of the following equation.

$$
\lim _{n \rightarrow \infty} o_{i}(K(n))=\infty \text {. }
$$

I present below a proof of $(R)$ which was found in January 1980. Later on some library research revealed to me that $(R)$ is a reformulation of a well-known combinatorial theorem discovered in 1930 by F. P. Ramsey [23]. This theorem has found numerous applications and has been generalised in many ways; see, e.g., [7, 8 and 12]. 
Note that $o_{i}(K(n))$ increases with $n$; so it is enough to prove that $o_{i}(K(n))$ can be equal to $k$ for only finitely many $n$.

Case $i=0$. If $o_{0}(K(n))=k$ choose a colouring $f$ of the vertices with $o_{0}(f)=k$; so $\operatorname{Im} f$ contains at most $k$ colours. One can find a set of vertices, at least $\{(n+1) / k\}$ in number, which is imaged by $f$ to the same colour $\alpha$ : so $k=o_{0}(f) \geqslant$ $f_{\alpha} \geqslant\{(n+1) / k\}$ which can hold for only finitely many $n$.

$(\mathrm{R})_{i-1}$ implies $(\mathrm{R})_{i}$. Let $k=o_{i}\left(K\left(n_{0}\right)\right)=o_{i}(K(n))$ where $n>n_{0}$ and choose a colouring $f$ of the $i$-simplices of $K(n)$ such that $o_{i}(f)=k$. Also choose a vertex $v$ of $K(n)$ and consider the $K(n-1)$ determined by the remaining vertices. We define a colouring $g$ of the $i-1$ simplices of $K(n-1)$ by setting $g(\sigma)=f(v . \sigma)$. We assert that $g_{\alpha} \leqslant n_{0} \forall \alpha \in \operatorname{Im}(g) \subseteq \operatorname{Im}(f)$. Otherwise there exists a $K\left(n_{0}\right) \subseteq K(n-1)$ all of whose $i-1$ simplices are given the same colour $\alpha$; using the definition of $g$ this implies $\left(f \mid \text { v. } K\left(n_{0}\right)\right)_{\alpha}=1+\left(f \mid K\left(n_{0}\right)\right)_{\alpha}$ and so leads to the contradiction $k=$ $o_{i}(f) \geqslant o_{i}\left(f \mid v \cdot K\left(n_{0}\right)\right) \geqslant 1+o_{i}\left(f \mid K\left(n_{0}\right)\right) \geqslant 1+k$. So

$$
o_{i-1}(K(n-1)) \leqslant o_{i-1}(g)=\sum_{\alpha \in \operatorname{Im} g} g_{\alpha} \leqslant n_{0} \frac{k}{i+1}
$$

since $\operatorname{Im}(f)$ - and so $\operatorname{Im}(g)$-does not contain more than $k /(i+1)$ colours (otherwise $\left.o_{i}(f)>(i+1) k /(i+1)=k\right)$. Now $(\mathrm{R})_{i-1}$ implies that this inequality can have only finitely many solutions in $n$.

(5.1.2) If $K$ is a simplicial complex and $X$ a triangulable space with $\operatorname{dim} X \geqslant$ $2(\operatorname{dim} K)+1$, then $X$ admits a triangulation $L$ such that $K \subseteq L$.

The proof below is similar to that of Exercise 25 of Grünbaum [13, p. 67].

Let $\Delta_{i}^{x}$ denote the simplicial complex determined by all the $i$-simplices $\sigma^{i} \subseteq$ $\{1,2, \ldots, x\}$. If $\operatorname{dim} K=i$, then $K$ is isomorphic to a subcomplex of $\Delta_{i}^{x}$ for all $x$ large enough. So it suffices to prove the result when $K=\Delta_{i}^{x}$. If $n \geqslant 2 i+1$, $x \geqslant n+2$, the cyclic triangulation $C_{n}^{x}$ of the $n$-sphere is $i$-neighbourly, i.e. $\Delta_{i}^{x} \subseteq C_{n}^{x}$. Choose any triangulation $M$ of $X^{n}$ and identify an $n$-simplex $\sigma^{n}$ of $M$ with the $n$-simplex $\{1,2, \ldots, n+1\}$ of $C_{n}^{x}$. Then $L=\left(M-\sigma^{n}\right) \cup\left(C_{n}^{x}-\sigma^{n}\right)$ is a triangulation of $X$ which contains $\Delta_{i}^{x}$.

(5.1.3) If $i$ is an integer $\geqslant 0$ and $X$ a triangulable space with $\operatorname{dim} X \geqslant 2 i+3$, then $\operatorname{ch}_{i}(X)=\infty$.

By (5.1.2) we see that for each integer $n \geqslant i+1,(K(n))_{i+1}$, the $i+1$ skeleton of $K(n)$, is a subcomplex of some triangulation $L_{n}$ of $X$. Since $\operatorname{ch}_{i}(X) \geqslant \operatorname{ch}_{i}\left(L_{n}\right) \geqslant$ $\operatorname{ch}_{i}\left((K(n))_{i+1}\right)=\operatorname{ch}_{i}(K(n))$, the result follows by $(5.1 .1)$.

We note that the result $\mathrm{ch}_{0}\left(S^{3}\right)=\infty$ goes back to Stäckel. (See Tietze [37].)

(5.2) If $M^{n}$ is a closed triangulable manifold with dimension $n \leqslant 2 i+2$, then the chromatic number $\operatorname{ch}_{i}\left(M^{n}\right)$ is finite. We hope to give a proof of this fact in another paper. (Note that in [25] this assertion was proved for all pseudomanifolds $M^{n}$, but under the stronger condition $n \leqslant i+2$.) The map colour theorem of Ringel et al. [24] and the four colour theorem of Appel and Haken [1] amount to the determination of the zeroth chromatic numbers $\mathrm{ch}_{0}\left(M^{2}\right)$ of closed 2-manifolds. (By using the four 
colour theorem we can prove that if $M^{n}, n \geqslant 3$, is a manifold, then $\operatorname{ch}_{n-2}\left(M^{n}\right) \leqslant 4$.) The codimension one chromatic numbers are much more easy to calculate. It is clear that $\operatorname{ch}_{0}\left(S^{1}\right)=3$; the remaining cases are taken care of by the following theorem.

(5.2.1) If $M^{n}$ is a closed triangulable manifold with dimension $n \geqslant 2$, then $\operatorname{ch}_{n-1}\left(M^{n}\right)=2$.

Let $K$ be any triangulation of $M^{n}$. Let $L$ be any subcomplex of $K$ which contains at least one $n$-simplex. We will prove, by induction on the number of $n-1$ simplices of $L$, that $\operatorname{ch}_{n-1}(L)=2$. Let $\sigma^{n-1}=v \cdot \theta^{n-2} \in L$. By the inductive hypothesis we can assign to the $n-1$ simplices of $L-\mathrm{St}_{L} \sigma$ two colours $\alpha$ and $\beta$ in such a way that not all faces of an $n$-simplex of $L$-St ${ }_{L} \sigma$ have the same colour. We can extend this "good" colouring of $L-\mathrm{St}_{L} \sigma$ to a good colouring of $L$ unless $L$ contains two $n$-simplices $u . \sigma^{n-1}$ and $w \cdot \sigma^{n-1}$; and all faces of $u . \sigma^{n-1}$ other than $\sigma$ have been ascribed the same colour (say $\alpha$ ) and all the faces of $w \cdot \sigma^{n-1}$ other than $\sigma$ have been ascribed the other colour $(\beta)$. In this "exceptional" case we will alter the colouring in $\left(L-\mathrm{St}_{L} \sigma\right) \cap\left(\mathrm{St}_{K} \theta\right)$ so as to obtain another good colouring of $L-\mathrm{St}_{L} \sigma$ which is no longer exceptional.

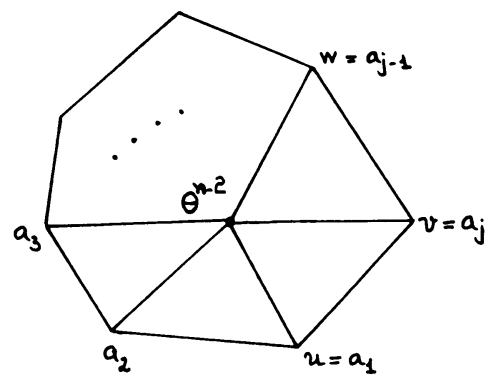

FIGURE 10

Let the vertices of the circle $\operatorname{Lk}_{K} \theta^{n-2}$ be $u=a_{1}, a_{2}, a_{3}, \ldots, a_{j-1}=w, a_{j}=v$ as in Figure 10. We change colour of $u \cdot \theta^{n-2}=a_{1} \cdot \theta^{n-2}$ from $\alpha$ to $\beta$. This can be objectionable only if $a_{1} \cdot a_{2} \cdot \theta^{n-2} \in L-\mathrm{St}_{L} \sigma$ and all faces of this $n$-simplex, other than $a_{1} \cdot \theta^{n-2}$ carry the colour $\beta$. If this is so we change colour of $a_{2} \cdot \theta^{n-2}$ from $\beta$ to $\alpha$. This can be objectionable only if $a_{2} \cdot a_{3} \cdot \theta^{n-2} \in L-\mathrm{St}_{L} \sigma$ and all faces of this $n$-simplex other than $a_{2} \cdot \theta^{n-2}$ carry the colour $\alpha \ldots$ Since $a_{j-1} \cdot a_{j} \cdot \theta^{n-2} \notin L-\mathrm{St}_{L} \sigma$ we will reach a stage when such a change in colour is no longer objectionable. The resultant colouring of $L-\mathrm{St}_{L} \sigma$ is both good and nonexceptional and so can be extended to $L$. This proves (5.2.1).

\section{Concluding remarks.}

(6.1) We discuss below some problems and conjectures concerning neighbourly triangulations.

(6.1.1) For any manifold $M$ we denote by $x_{M}$ the least number of vertices required to triangulate it. The reader can verify that, for neighbourly 2 -manifolds, this agrees 
with the notation of (2.1.2). Thus, in general, the problem of calculating $x_{M}$ is forbiddingly hard: already, for closed 2-manifolds, it is equivalent to the map colour theorem of Ringel et al. [24].

However it should be interesting to find methods which would allow one to calculate $x_{M}$ for a large enough class of 3-manifolds; e.g. for all handelbodies $M^{3}$ for which $\partial M^{3}$ is neighbourly. Such methods would probably also lead to a clearer understanding of the map colour theorem. Note that $(4.2 .5)(\mathrm{d})$ shows that if $M^{3}=D^{2} \times S^{1}$, then $x_{M}=7$; we refer the reader to Altshuler [30] (see also [31]) for the proof of the fact that if $M^{3}=S^{2} \times S^{1}$, then $x_{M}=10$.

We expect that there exist closed connected 3-manifolds $M^{3}$ for which the number $\nu_{M}$ of (3.2.1) is strictly bigger than $x_{M}$; however no such example has yet been found.

(6.1.2) Upper and lower bounds. We conjecture that for any closed connected manifold $M^{n}$ and any integer $x \geqslant x_{M}$ there exist triangulations $K_{x}$ and $K^{x}$ such that if $K$ is a triangulation of $M^{n}$ with $\alpha_{0}(K)=x$, then $\alpha_{i}\left(K_{x}\right) \leqslant \alpha_{i}(K) \leqslant \alpha_{i}\left(K^{x}\right)$ for all $i, 1 \leqslant i \leqslant n$. Furthermore $K_{x}$ can be defined inductively as follows: $K_{x_{M}}$ is a triangulation with $x_{M}$ vertices having the least number of $n$-simplices, and $K_{x+1}$ is obtained by deriving an $n$-simplex of $K_{x}$. If, in addition, the homotopy groups $\pi_{i}\left(M^{n}\right), 0 \leqslant i<[(n-1) / 2]$, are trivial, then for all $x$ sufficiently big, $K^{x}$ will be an [(n-1)/2]-neighbourly triangulation of $M^{n}$.

We note that if $M^{n}=S^{n}$, then the conjectured lower bound $\alpha_{i}\left(K_{x}\right)$ agrees with that on p. 183 of Grünbaum [13]; also, in this case, Stanley [28] has proved the second inequality with $K^{x}=C_{n}^{x}$ for each $x \geqslant n+2$. It seems that the above lower bound conjecture has been proved for $S^{n}$ by D. Barnette. See [32, p. 354 and 33].

(6.1.3) The last part of conjecture (6.1.2) implies, in particular, that we expect the homotopy groups $\pi_{i}(M), 0 \leqslant i<[(n-1) / 2]$, to be the only obstructions to the existence of $[(n-1) / 2]$-neighbourly triangulations.

If $d>[(n-1) / 2]$ then $\pi_{i}\left(M^{n}\right)=0,0 \leqslant i<d$, does not suffice to ensure the existence of $d$-neighbourly triangulations. This is already indicated for $n=2$ by (2.1.1); we will now prove that not every simply connected 4-manifold $M^{4}$ admits a 2-neighbourly triangulation. If $K$ is any triangulation of $M^{4}$ then we have the Dehn-Sommerville equations $2 \alpha_{3}(K)=5 \alpha_{4}(K), 2 \alpha_{1}(K)=3 \alpha_{2}(K)-6 \alpha_{3}(K)+$ $10 \alpha_{4}(K)$ and $\mathfrak{X}\left(M^{4}\right)=\alpha_{0}(K)-\alpha_{1}(K)+\alpha_{2}(K)-\alpha_{3}(K)+\alpha_{4}(K)$ : see (6.1.4). If $K$ is 2-neighbourly and has $x$ vertices $(x \geqslant 6)$, then $\alpha_{0}(K)=x, \alpha_{1}(K)=\left(\begin{array}{l}x \\ 2\end{array}\right)$ and $\alpha_{2}(K)=\left(\begin{array}{l}x \\ 3\end{array}\right)$. Substituting these values in the above equations and simplifying we see that $M^{4}$ can admit a 2-neighbourly triangulation only if the Euler characteristic $\mathfrak{X}\left(M^{4}\right)$ is such that $60 \mathfrak{X}\left(M^{4}\right)=x\left(x^{2}-15 x+74\right)$ for some integer $x \geqslant 6$. The connected sum $\left(S^{2} \times S^{2}\right) \#\left(\mathbf{C} P^{2}\right)$ is a simply connected 4-manifold with Euler characteristic 5; further it is easily seen that $300=x\left(x^{2}-15 x+74\right)$ has no integer solutions $\geqslant 6$.

However we do conjecture that a closed 3-manifold admits a 2-neighbourly triangulation if and only if it is simply connected. This is merely a simple reformulation of the celebrated Poincaré conjecture: if a closed simply connected 3-manifold $M^{3}$ admits a 2-neighbourly triangulation with $x$ vertices then $x-\left(\begin{array}{l}x \\ 2\end{array}\right)+\left(\begin{array}{l}x \\ 3\end{array}\right)-\frac{1}{2}\left(\begin{array}{l}x \\ 3\end{array}\right)$ $=0$; this cubic has the roots 0,4 and 5; so $M^{3}$ has a triangulation with 5 vertices 
and must be homeomorphic to $S^{3}$. Thus Poincare's conjecture is the natural analogue of the 'colouring theorem' (2.1.1).

The above discussion indicates why we expect the general problem of finding homotopy theoretic conditions equivalent to the existence of $d$-neighbourly triangulations to be very hard when $d>[(n-1) / 2]$.

(6.1.4) $A$ functional equation. For any simplicial complex $K$ we define $\alpha_{K}(z)=1$ $-\alpha_{0}(K) . z+\alpha_{1}(K) \cdot z^{2}-\alpha_{2}(K) \cdot z^{3}+\cdots$. If $K$ triangulates a closed manifold $M^{n}$, then the link of each $i$-simplex has the homology groups of $S^{n-i-1}$ (see, e.g., [35, Proposition 1.2]) and so has Euler characteristic $1+(-1)^{n-i-1}$. From this fact one gets the equations

$$
\left(1+(-1)^{n-i-1}\right) \alpha_{i}=\sum_{j \geqslant 0}(-1)^{j}\left(\begin{array}{c}
j+i+2 \\
i+1
\end{array}\right) \alpha_{j+i+1} .
$$

These equations are due to Dehn [5], Sommerville [27] and Klee [15]. They can be written in a concise way as

$$
(-1)^{n} \alpha_{K}(z)+\alpha_{K}(1-z)=1+(-1)^{n}-\mathscr{X}\left(M^{n}\right) .
$$

This functional equation is given in I. G. Macdonald [17].

An easy consequence of $(\mathrm{F})$ is that if $K_{1}$ (resp. $\left.K_{2}\right)$ triangulates $M_{1}^{n}\left(\right.$ resp. $\left.M_{2}^{n}\right)$ and $\mathscr{X}\left(M_{1}^{n}\right)=\mathscr{X}\left(M_{2}^{n}\right)$, then $\alpha_{i}\left(K_{1}\right)=\alpha_{i}\left(K_{2}\right), 1 \leqslant i \leqslant[(n-1) / 2]$ implies $\alpha_{i}\left(K_{1}\right)=$ $\alpha_{i}\left(K_{2}\right)$ for all $i$. So if $K^{x}$ is an [(n-1)/2]-neighbourly triangulation of $M^{n}$ (this can happen only if $\left.\pi_{i}\left(M^{n}\right)=0,0 \leqslant i<(n-1) / 2\right)$ and $\mathscr{X}\left(M^{n}\right)=\mathscr{X}\left(S^{n}\right)$, then we must have $\alpha_{i}\left(K^{x}\right)=\alpha_{i}\left(C_{n}^{x}\right)$ for all $i$. This suggests a conjecture: if $K$ is a triangulation of $M^{n}$ with $x$ vertices and $\mathscr{X}\left(M^{n}\right)=\mathscr{X}\left(S^{n}\right)$ then $\alpha_{i}(K) \leqslant \alpha_{i}\left(C_{n}^{x}\right)$ for all $i$. However the homotopy groups $\pi_{i}\left(M^{n}\right), 0 \leqslant i<[(n-1) / 2]$, may not be trivial; thus these upper bounds will not be, in general, the best possible.

(6.1.5) $A$ Riemann hypothesis. If $K$ triangulates the closed manifold $M^{n}$ and $\mathcal{X}\left(M^{n}\right)=\mathscr{X}\left(S^{n}\right)$, then the functional equation (F) shows that the roots of the polynomial $\alpha_{K}(z)$ are symmetrically situated with respect to the real axis and the line $\operatorname{Re} z=\frac{1}{2}$. We will say that the Riemann hypothesis holds for $K$ if all the roots lie on these two lines. The problem is to characterize all triangulations of $M^{n}$ for which the Riemann hypothesis holds.

EXAMPLES. (1) The Riemann hypothesis holds for the minimal triangulation of $S^{n}$. In fact now $\alpha_{K}(z)=(1-z)^{n+2}-(-1)^{n+2} z^{n+2}$; so $z$ is a root only if $w=z /(1-z)$ is a root of $w^{n+2}=(-1)^{n+2}$; hence $|z /(1-z)|=1$ and so $\operatorname{Re} z=\frac{1}{2}$.

(2)(a) Any closed 3-manifold admits an infinite number of triangulations for which the Riemann hypothesis holds. If $K$ is a triangulation of the closed manifold $M^{3}$ and $\alpha_{0}(K)=m, \alpha_{1}(K)=n$, then $\alpha_{K}(z)=1-m z+n z^{2}-2(n-m) z^{3}+(n-m) z^{4}$. Put $z=\frac{1}{2}+z^{\prime}$; in the resulting polynomial the coefficients of $z^{\prime}$ and $\left(z^{\prime}\right)^{3}$ will be zero. Now let $w=\left(z^{\prime}\right)^{2}$; we will get

$$
(n-m) w^{2}+((3 m-n) / 2) w+(16-5 m+n) / 16 .
$$

The Riemann hypothesis holds for $K$ iff the roots of this quadratic are real i.e. iff $((3 m-n) / 2)^{2}-(16-5 m+n)(n-m) / 4 \geqslant 0$, i.e. iff $m^{2}+4 m \geqslant 4 n$. Now for each $x \geqslant x_{M}$ let $K_{x}$ be as in (6.1.2): so $m=x$ and $n=c+4\left(x-x_{M}\right)$ where 
$c=\alpha_{1}\left(K_{x_{M}}\right)$. Thus the Riemann hypothesis holds for $K_{x}$ iff $x^{2}+4 x \geqslant 4 c+16 x-$ $16 x_{M}$ which will be true for all $x$ sufficiently large. (b) Any closed 3-manifold admits an infinite number of triangulations for which the Riemann hypothesis does not hold. By (3.2.1) there exists an integer $\nu_{M}$ such that for all $x \geqslant \nu_{M}, M$ admits a neighbourly triangulation $K^{x}$ having $x$ vertices. If $x \geqslant 7$ the Riemann hypothesis will not hold for $K^{x}$. To see this note that $m=x, n=\left(\begin{array}{l}x \\ 2\end{array}\right)$ and so the requirement $m^{2}+4 m \geqslant 4 n$, i.e. $x^{2}+4 x \geqslant 4\left(\begin{array}{l}x \\ 2\end{array}\right)$ reads $x \leqslant 6$.

We expect the general situation to be similar to that shown by Example (2) above: if $M^{n}$ is a closed manifold and $\mathfrak{X}\left(M^{n}\right)=\mathscr{X}\left(S^{n}\right)$, then the Riemann hypothesis will hold (resp. will not hold) for all triangulations $K_{x}$ (resp. $K^{x}$ ) provided $x$ is sufficiently large. Here $K_{x}$ and $K^{x}$ are as in (6.1.2).

(6.1.6) We conjecture that every orientable connected 3-manifold with connected nonempty boundary admits an order-orientable triangulation: compare (4.2.5)(c). It is possible that such manifolds in fact admit neighbourly order-orientable triangulations; such triangulations would generalise the triangulation $D_{3}^{x}$ of (4.2.5)(a).

(6.2) The following historical and bibliographical remarks regarding monotone sequences may be useful to the reader.

(6.2.1) Infinite monotone sequences. In his paper of 1914, Hjelmslev [14] proved the following theorem. If $a_{1}, a_{2}, \ldots$ is an infinite bounded monotone sequence of $A^{n}$, then it has a unique limit point $a$ and for each $p, 1 \leqslant p \leqslant n$, a $p$-dimensional affine subspace determined by $p+1$ entries of this sequence tends towards a $p$-dimensional affine subspace through $a$; conversely the existence of these limits guarantees that a given sequence of $A^{n}$ is eventually monotone.

(6.2.2) A directed arc in $A^{n}$ is called a monotone curve if any $n+1$ of its points are affinely independent and determine the same orientation of $A^{n}$. This terminology is also due to Hjelmslev [14]. But it is important to note that already in $1886 \mathrm{~A}$. A. Markov [18] had proved a beautiful theorem which solved the following problem when the curve in question is differentiable and monotone.

The Chebyshev-Markov moment problem. Let $L=\overparen{a c b}$ be an arc in $A^{n}$ and let $p$ be a point lying in the convex hull of $L$; it is required to find inf $\mu(\widehat{a c})$ and $\sup \mu(\widehat{a c})$ as $\mu$ varies over all (normalised, positive, Radon) measures on $L$ having centre of gravity $p$.

A concise history of the above problem and an elegant presentation of Markov's theorem is given in Krein [16]. We remark that people working in the theory of moments usually refer to monotone curves as "Chebyshev (or Tchebycheff or $T$-) systems"; this terminology was coined by S. N. Bernstein in 1934 (see Krein [16]).

(6.2.3) It is easy to see that an arc of $A^{n}$ is monotone if and only if every codimension one affine subspace of $A^{n}$ cuts it in $\leqslant n$ points; for this reason some people refer to monotone curves as "curves of order $\leqslant n$ " (see, e.g., McMullen and Shephard [20, p. 82]). This reformulation suggets that a homeomorphism of an $m$-dimensional manifold $M^{m}$ into $A^{n}$ be called a monotone imbedding if every codimension $m$ affine subspace cuts it in $\leqslant n-m+1$ points. It is easy to give numerous examples of such imbeddings.

(6.2.4) One can show that any (finite) monotone sequence can be extrapolated to a monotone curve. (This result resembles Theorem 2.1 of Fr. Fabricius-Bjerre [9].) 
Using this lemma one can also prove (4.3.2) in the usual way as on pp. 84-85 of McMullen and Shephard [20].

(6.2.5) If $f: S^{1} \rightarrow A^{n}$ is a monotone imbedding, then $f\left(S^{1}\right) \subseteq A^{n}$ is called a closed monotone curve. For example one has the curve $L \subseteq \mathbf{R}^{2 n}$ given by $\theta \mapsto$ $(\cos \theta, \sin \theta, \ldots, \cos n \theta, \sin n \theta)$.

Carathéodory's problem. What are the conditions on a point $c=\left(c_{1}, c_{2}, \ldots, c_{n}\right) \in$ $\mathbf{C}^{n}$ so that there exists, on the open unit disk, a holomorphic function with positive real part whose power series expansion reads $\frac{1}{2}+c_{1} z+c_{2} z^{2}+\cdots+c_{n} z^{n}+\cdots$ ?

This is an easy problem which was raised and settled by Carathéodory [3] in 1907: if one identifies $\mathbf{C}^{n}$ with $\mathbf{R}^{2 n}$ under $\left(z_{1}, z_{2}, \ldots, z_{n}\right) \mapsto\left(\operatorname{Re} z_{1}, \operatorname{Im} z_{1}, \ldots, \operatorname{Re} z_{n}, \operatorname{Im} z_{n}\right)$, the necessary and sufficient condition is that $\bar{c}$ belong to the convex hull of the closed monotone curve $L \subseteq \mathbf{R}^{2 n}$. Note that this result can obviously also be stated as a theorem in the theory of moments: the necessary and sufficient condition is that there exists a measure on $L$ whose centre of gravity is $\bar{c}$. There are some problems in complex function theory (e.g. the celebrated "Bieberbach conjecture') which are much deeper than Carathéodory's problem but yet have the same flavour. These problems are intimately related to parallel questions in the theory of moments and in all these considerations the concept of monotonicity has played a key role. See e.g. [26 and 6].

In 1911 Carathéodory [4] published a sequel to [3] in which he indicated that for any finite set $X$ of $L \subseteq \mathbf{R}^{2 n}$ with $x \geqslant 2 n+1$ the simplicial complex $K_{X}$ is $n-1$ neighbourly; however the combinatorial nature of $K_{X}$, e.g. the isomorphism $K_{X} \cong$ $C_{2 n-1}^{x}$, was understood only after the work of Gale $[10,11]$ and Motzkin [22].

\section{REFERENCES}

1. K. Appel and W. Haken, Every planar map is four colorable, Bull. Amer. Math. Soc. 82 (1976), $711-712$.

2. R. H. Bing, Some aspects of the topology of 3-manifolds related to the Poincare conjecture, Lectures on Modern Mathematics, Vol. II, edited by T. L. Saaty, Wiley, New York, 1964, pp. 93-128.

3. C. Carathéodory, Über den Variabilitätsbereich der Koeffizienten von Potenzreihen, die gegebene Werte nicht annehemen, Math. Ann. 64 (1907), 95-115.

4. _ Über den Variabilitätsbereich der Fourierschen Konstanten von positiven harmonischen Funktionen, Rend. Circ. Mat. Palermo 32 (1911), 193-217.

5. M. Dehn, Die Eulersche Formel in Zusammenhang mit dem Inhalt in der nicht-Euklidischen Geometrie, Math. Ann. 61 (1905), 561-586.

6. W. F. Donoghue, Jr., Monotone matrix functions and analytic continuation, Springer-Verlag, Berlin and New York, 1974.

7. P. Erdös and R. Rado, A combinatorial theorem, J. London Math. Soc. 25 (1950), 249-255.

8. __ A partition calculus in set theory, Bull. Amer. Math. Soc. 62 (1956), 427-489.

9. Fr. Fabricius-Bjerre, On polygons of order $n$ in projective $n$-space, with an application to strictly convex curves, Math. Scand. 10 (1962), 221-229.

10. D. Gale, Neighboring vertices on a convex polyhedron, Linear Inequalities and Related Topics (Editors H. W. Kuhn and A. W. Tucker), Princeton Univ. Press, Princeton, N.J., 1956, pp. 255-263.

11. __ Neighborly and cyclic polytopes, Convexity, Proc. Sympos. Pure Math., vol. 7, Amer. Math. Soc., Providence, R.I., 1963, pp. 225-232.

12. R. L. Graham and B. L. Rothschild, Some recent developments in Ramsey theory, Combinatorics, Proc. NATO Advanced Study Inst. (Editors M. Hall Jr. and J. H. van Lint), Reidel, Dordrecht, 1974, pp. 261-276.

13. B. Grünbaum, Convex polytopes, Wiley, New York, 1967.

14. J. Hjelmslev, Introduction à la théorie des suites monotones, Dan. Vid. Selsk. Forh. 1 (1914), 1-74. 
15. V. Klee, A combinatorial analogue of Poincaré's duality theorem, Canad. J. Math. 16 (1964), $517-531$.

16. M. G. Krein, The ideas of P. L. Čebyšev and A. A. Markov in the theory of limiting values of integrals and their further development, Amer. Math. Soc. Transl. (2) 12 (1959), 1-121 (= Uspehi Mat. Nauk 6 (1951), 3-120).

17. I. G. Macdonald, Polynomials associated with finite cell complexes, J. London Math. Soc. 4 (1971), $181-192$.

18. (A. A. Markov), Extrait d'une lettre adressé à M. Hermite, Ann. Sci. École Norm. Sup. 3 (1886), $81-88$.

19. P. McMullen, The number of faces of simplicial polytopes, Israel J. Math. 9 (1971), 559-570.

20. P. McMullen and G. C. Shephard, Convex polytopes and the upper bound conjecture, Cambridge Univ. Press, Cambridge, 1971.

21. E. E. Moise, Affine structures in 3-manifolds. V: The triangulation theorem and Hauptvermutung, Ann. of Math. (2) 56 (1952), 96-114.

22. T. S. Motzkin, Comonotone curves and polyhedra, Abstract 111, Bull. Amer. Math. Soc. 63 (1957), 35.

23. F. P. Ramsey, On a problem of formal logic, Proc. London Math. Soc. (2) 30 (1930), 264-286.

24. G. Ringel, Map color theorem, Springer-Verlag, Berlin and New York, 1974.

25. K. S. Sarkaria, On coloring manifolds, Illinois J. Math. 25 (1981), 464-469.

26. J. A. Shohat and J. D. Tamarkin, The problem of moments, Amer. Math. Soc., Providence, R.I., 1943.

27. D. M. Y. Sommerville, The relations connecting the angle sums and volume of a polytope in space of $n$ dimensions, Proc. Roy. Soc. London Ser. A 115 (1927), 103-119.

28. R. P. Stanley, The upper bound conjecture and Cohen-Macaulay rings, Stud. Appl. Math. 54 (1975), $135-142$.

29. B. Uhrin, problem proposed in the Fifth Hungarian Colloquium, Keszthely, 1976, Colloq. Math. Soc. János Bolyai 18, North-Holland, Amsterdam, 1978.

30. A. Altshuler, Neighborly 4-polytopes and neighborly combinatorial 3-manifolds with ten vertices, Canad. J. Math. 29 (1977), 400-420.

31. A. Altshuler and L. Steinberg, An enumeration of combinatorial 3-manifolds with 9 vertices, Discrete Math. 16 (1976), 91-108.

32. D. Barnette, $A$ proof of the lower bound conjecture for convex polytopes, Pacific J. Math. 46 (1973).

33. Graph theorems for manifolds, Israel J. Math. 16 (1973), 62-72.

34. A. Császár, A polyhedron without diagonals, Acta Sci. Math. (Szeged) 13 (1949-50), 140-142.

35. D. E. Galewski and R. J. Stern, Classification of simplicial triangulations of topological manifolds, Ann. of Math. (2) 111 (1980), 1-34.

36. A. F. Möbius, Gesammelte Werke, Vol. 2, Leipzig, 1886, pp. 552-553.

37. H. Tietze, Über das Problem der Nachbargebiete im Raum, Monatsh. Math. Physik 16 (1905), 211-216.

38. D. W. Walkup, The lower bound conjecture for 3- and 4-manifolds, Acta Math. 125 (1970), 75-107.

213, 16A, Chandigarh 160016, INDIA 